\title{
NEW UNIFORM AND ASYMPTOTIC UPPER BOUNDS ON THE TENSOR RANK OF MULTIPLICATION IN EXTENSIONS OF FINITE FIELDS
}

\author{
JULIA PIELTANT AND HUGUES RANDRIAM
}

\begin{abstract}
We obtain new uniform upper bounds for the tensor rank of the multiplication in the extensions of the finite fields $\mathbb{F}_{q}$ for any prime power $q$; moreover, these uniform bounds lead to new asymptotic bounds as well. In addition, we also give purely asymptotic bounds which are substantially better by using a family of Shimura curves defined over $\mathbb{F}_{q}$, with an optimal ratio of $\mathbb{F}_{q^{t}}$-rational places to their genus, where $q^{t}$ is a square.
\end{abstract}

\section{INTRODUCTION}

1.1. Tensor rank of multiplication. Let $K$ be a field and let $\mathcal{A}$ be a finitedimensional $K$-algebra. We denote by $m_{\mathcal{A}}$ the multiplication map of $\mathcal{A}$. It can be seen as a $K$-bilinear map from $\mathcal{A} \times \mathcal{A}$ into $\mathcal{A}$, or equivalently, as a linear map from the tensor product $\mathcal{A} \otimes \mathcal{A}$ over $K$ into $\mathcal{A}$. One can also represent it by a tensor $t_{\mathcal{A}} \in \mathcal{A}^{\star} \otimes \mathcal{A}^{\star} \otimes \mathcal{A}$ where $\mathcal{A}^{\star}$ denotes the dual of $\mathcal{A}$ over $K$. Hence the product of two elements $x$ and $y$ of $\mathcal{A}$ is the convolution of this tensor with $x \otimes y \in \mathcal{A} \otimes \mathcal{A}$. If

$$
t_{\mathcal{A}}=\sum_{l=1}^{\lambda} a_{l} \otimes b_{l} \otimes c_{l}
$$

where $a_{l} \in \mathcal{A}^{\star}, b_{l} \in \mathcal{A}^{\star}, c_{l} \in \mathcal{A}$, then

$$
x \cdot y=\sum_{l=1}^{\lambda} a_{l}(x) b_{l}(y) c_{l} .
$$

Every expression (2) is called a bilinear multiplication algorithm $\mathcal{U}$ for $\mathcal{A}$ over $K$. The integer $\lambda$ is called the bilinear complexity $\mu(\mathcal{U})$ of $\mathcal{U}$.

Let us set

$$
\mu_{K}(\mathcal{A})=\min _{\mathcal{U}} \mu(\mathcal{U}),
$$

where $\mathcal{U}$ is running over all bilinear multiplication algorithms for $\mathcal{A}$ over $K$.

Then $\mu_{K}(\mathcal{A})$ corresponds to the minimum possible number of summands in any tensor decomposition of type (11), which is the rank of the tensor of multiplication in $\mathcal{A}$ over $K$. The tensor $\operatorname{rank} \mu_{K}(\mathcal{A})$ is also called the bilinear complexity of multiplication in $\mathcal{A}$ over $K$.

Received by the editor May 22, 2013 and, in revised form, November 22, 2013.

2010 Mathematics Subject Classification. Primary 14H05; Secondary 11Y16, 12E20.

Key words and phrases. Algebraic function field, tower of function fields, tensor rank, algorithm, finite field. 
When the decomposition (11) is symmetric, i.e., $a_{l}=b_{l}$ for all $l=1, \ldots, \lambda$, we say that the corresponding algorithm $\mathcal{U}$ is a symmetric bilinear multiplication algorithm. If we focus on such algorithms, then the corresponding complexity is called the symmetric bilinear complexity of multiplication in $\mathcal{A}$ over $K$ and we set

$$
\mu_{K}^{\mathrm{sym}}(\mathcal{A})=\min _{\mathcal{U}^{\mathrm{sym}}} \mu\left(\mathcal{U}^{\mathrm{sym}}\right),
$$

with $\mathcal{U}^{\text {sym }}$ running over all symmetric bilinear multiplication algorithms for $\mathcal{A}$ over $K$. Note that one has

$$
\mu_{K}(\mathcal{A}) \leq \mu_{K}^{\mathrm{sym}}(\mathcal{A})
$$

In this work we will be mainly interested in the case where $K=\mathbb{F}_{q}$ is the finite field with $q$ elements and $\mathcal{A}=\mathbb{F}_{q^{n}}$ is the extension field of degree $n$ of $\mathbb{F}_{q}$. We then set

$$
\mu_{q}(n)=\mu_{\mathbb{F}_{q}}\left(\mathbb{F}_{q^{n}}\right)
$$

However, for technical reasons we will also need the quantities

$$
\mu_{q}(m, l)=\mu_{\mathbb{F}_{q}}\left(\mathbb{F}_{q^{m}}[t] /\left(t^{l}\right)\right)
$$

so that $\mu_{q}(n)=\mu_{q}(n, 1)$.

Similarly, we set $\mu_{q}^{\mathrm{sym}}(n)=\mu_{\mathbb{F}_{q}}^{\mathrm{sym}}\left(\mathbb{F}_{q^{n}}\right)$ and $\mu_{q}^{\mathrm{sym}}(m, l)=\mu_{\mathbb{F}_{q}}^{\mathrm{sym}}\left(\mathbb{F}_{q^{m}}[t] /\left(t^{l}\right)\right)$.

1.2. Notations. Let $F / \mathbb{F}_{q}$ be an algebraic function field of one variable of genus $g$, with constant field $\mathbb{F}_{q}$, associated to a curve $X$ defined over $\mathbb{F}_{q}$. For any place $P$ we define $F_{P}$ to be the residue class field of $P$ and $\mathcal{O}_{P}$ its valuation ring. Every element $t \in P$ such that $P=t \mathcal{O}_{P}$ is called a local parameter for $P$ and we denote by $v_{P}$ a discrete valuation associated to the place $P$ of $F / \mathbb{F}_{q}$. Recall that this valuation does not depend on the choice of the local parameter. Let $f \in F \backslash\{0\}$, we denote by $(f):=\sum_{P} v_{P}(f) P$ where $P$ is running over all places in $F / \mathbb{F}_{q}$, the principal divisor of $f$. If $\mathcal{D}$ is a divisor then $\mathcal{L}(\mathcal{D})=\left\{f \in F / \mathbb{F}_{q} ; \mathcal{D}+(f) \geq 0\right\} \cup\{0\}$ is a vector space over $\mathbb{F}_{q}$ whose dimension $\operatorname{dim} \mathcal{D}$ is given by the Riemann-Roch Theorem. The degree of a divisor $\mathcal{D}=\sum_{P} a_{P} P$ is defined by $\operatorname{deg} \mathcal{D}=\sum_{P} a_{P} \operatorname{deg} P$ where $\operatorname{deg} P$ is the dimension of $F_{P}$ over $\mathbb{F}_{q}$. The order of a divisor $\mathcal{D}=\sum_{P} a_{P} P$ at $P$ is the integer $a_{P}$ denoted by $\operatorname{ord}_{P} \mathcal{D}$. The support of a divisor $\mathcal{D}$ is the set $\operatorname{supp} \mathcal{D}$ of the places $P$ such that $\operatorname{ord}_{P} \mathcal{D} \neq 0$. Two divisors $\mathcal{D}$ and $\mathcal{D}^{\prime}$ are said to be equivalent if $\mathcal{D}=\mathcal{D}^{\prime}+(x)$ for an element $x \in F \backslash\{0\}$.

We denote by $B_{k}\left(F / \mathbb{F}_{q}\right)$ the number of places of degree $k$ of $F / \mathbb{F}_{q}$ and by $g\left(F / \mathbb{F}_{q}\right)$ the genus of $F / \mathbb{F}_{q}$.

1.3. Known results. The bilinear complexity $\mu_{q}(n)$ of the multiplication in the $n$-degree extension of a finite field $\mathbb{F}_{q}$ is known for certain values of $n$. In particular, S. Winograd [21] and H. de Groote [15] have shown that this complexity is $\geq 2 n-1$, with equality holding if and only if $n \leq \frac{1}{2} q+1$. Moreover, in this case one has $\mu_{q}^{\mathrm{sym}}(n)=\mu_{q}(n)$. Using the principle of the D.V. and G.V. Chudnovsky algorithm [14] applied to elliptic curves, M.A. Shokrollahi has shown in [19] that the symmetric bilinear complexity of multiplication is equal to $2 n$ for $\frac{1}{2} q+1<n<\frac{1}{2}(q+1+\epsilon(q))$ where $\epsilon$ is the function defined by

$$
\epsilon(q)=\left\{\begin{array}{l}
\text { the greatest integer } \leq 2 \sqrt{q} \text { prime to } q, \text { if } q \text { is not a perfect square } \\
2 \sqrt{q}, \text { if } q \text { is a perfect square. }
\end{array}\right.
$$

Moreover, U. Baum and M.A. Shokrollahi have succeeded in [11] to construct effective optimal algorithms of the Chudnovsky type in the elliptic case. 
Recently in [1, 2], 9], 7], 6], 4] and [3] the study made by M.A. Shokrollahi has been generalized to algebraic function fields of genus $g$.

Let us recall that the original algorithm of D.V. and G.V. Chudnovsky introduced in [14] leads to the following theorem proved in [1]:

Theorem 1.1. Let $q=p^{r}$ be a power of the prime $p$. The symmetric tensor rank $\mu_{q}^{\mathrm{sym}}(n)$ of multiplication in any finite field $\mathbb{F}_{q^{n}}$ is linear with respect to the extension degree; more precisely, there exists a constant $C_{q}$ such that

$$
\mu_{q}^{\mathrm{sym}}(n) \leq C_{q} n .
$$

Moreover, one can give explicit values for $C_{q}$ :

Proposition 1.2. The best known values for the constant $C_{q}$ defined in the previous theorem are:

$$
C_{q}=\left\{\begin{array}{lll}
\text { if } q=2 & \text { then } 22 & {[13] \text { and }[8]} \\
\text { else if } q=3 & \text { then } 27 & {[1]} \\
\text { else if } q=p \geq 5 & \text { then } 3\left(1+\frac{4}{q-3}\right) & {[4]} \\
\text { else if } q=p^{2} \geq 25 & \text { then } 2\left(1+\frac{2}{\sqrt{q}-3}\right) & {[4]} \\
\text { else if } q=p^{2 k} \geq 16 & \text { then } 2\left(1+\frac{p}{\sqrt{q}-3}\right) & {[2]} \\
\text { else if } q \geq 16 & \text { then } 3\left(1+\frac{2 p}{q-3}\right) & {[9],[7] \text { and }[6]} \\
\text { else if } q>3 & \text { then } 6\left(1+\frac{p}{q-3}\right) & {[2 .}
\end{array}\right.
$$

In order to obtain these good estimates for the constant $C_{q}, \mathrm{~S}$. Ballet has given in [1] some easy to verify conditions allowing the use of the D.V. and G.V. Chudnovsky algorithm. Then S. Ballet and R. Rolland have generalized in [9] the algorithm using places of degree one and two.

Recently, various generalizations of this algorithm were introduced in 13 and then in [18. We will use the version that can be found in [18, Proposition 5.7] and which, expressed in the language of function fields, reads as follows:

Theorem 1.3. Let $F / \mathbb{F}_{q}$ be an algebraic function field of genus $g \geq 2$, and let $m, l \geq 1$ be two integers.

Suppose that $F$ admits a place of degree $m$ (a sufficient condition for this is $\left.2 g+1 \leq q^{(m-1) / 2}\left(q^{1 / 2}-1\right)\right)$.

Now consider a collection of integers $n_{d, u} \geq 0$ (for $d, u \geq 1$ ), such that almost all of them are zero, and that for any $d$,

$$
\sum_{u} n_{d, u} \leq B_{d}\left(F / \mathbb{F}_{q}\right)
$$

Suppose the following assumption is satisfied:

$$
\sum_{d, u} n_{d, u} d u \geq 2 m l+3 e+g-1
$$

where the constant $e$ is defined as $e=2$ if $q=2, e=1$ if $q=3,4,5$, and $e=0$ if $q \geq 7$. Then we have

$$
\mu_{q}(m, l) \leq \sum_{d, u} n_{d, u} \mu_{q}(d, u)
$$

Intuitively, the algorithm works as follows: if $x, y$ are two elements in $\mathbb{F}_{q^{m}}[t] /\left(t^{l}\right)$ to be multiplied, we lift them to functions $f_{x}, f_{y}$ in some well-chosen Riemann-Roch spaces of $F$, we evaluate these functions at various places of $F$ with multiplicities 
(more precisely, $n_{d, u}$ is the number of places of degree $d$ used with multiplicity $u$ ), we multiply these values locally, and then we interpolate to find the product function $f_{x} f_{y}$, from which the product $x y$ is deduced.

Note that this algorithm is not necessarily symmetric since $f_{x}$ and $f_{y}$ can be lifted in two different Riemann-Roch spaces; so we obtain bounds for $\mu_{q}(m, l)$, and not for $\mu_{q}^{\mathrm{sym}}(m, l)$.

1.4. New results established in this paper. In Section 2, we describe a general method to obtain new uniform bounds for the bilinear complexity of multiplication, by applying the algorithm recalled in Theorem 1.3 on towers of function fields which satisfy some properties.

In Section 3, we recall some results about a completed Garcia-Stichtenoth tower [16] studied in [2] and about the Garcia-Stichtenoth tower introduced in [17]. For both towers, we study some of their properties which will be useful in Section 4, to apply the general method on these towers. By doing so, we obtain in Section 4, new uniform bounds on the (asymmetric) bilinear complexity of multiplication in extensions of $\mathbb{F}_{2}$, of $\mathbb{F}_{q^{2}}$ and $\mathbb{F}_{q}$ for any prime power $q \geq 4$ and of $\mathbb{F}_{p^{2}}$ and $\mathbb{F}_{p}$ for any prime $p \geq 3$, which are the best ones currently known.

Last, in Section 5, we turn to the asymptotics of the bilinear complexity as the degree of the extension goes to infinity. In some cases, the asymptotics of our uniform bounds already improve on previously known results. But then we also present some (non-uniform) bounds with even better asymptotics, which appear to establish a new present state of the art.

\section{General algorithm USED in this PAPeR}

Lemma 2.1. Let $d$ be a positive integer. For any integer $0<j \leq d$ such that $j<\frac{1}{2}(q+1+\epsilon(q))$ if $q \geq 4$, or $j \leq \frac{1}{2} q+1$ if $q \in\{2,3\}$, one has

$$
\frac{\mu_{q}^{\mathrm{sym}}(j)}{j} \leq \frac{\mu_{q}^{\mathrm{sym}}(d)}{d}
$$

where we recall from Section 1.3 that

$$
\epsilon(q)=\left\{\begin{array}{l}
\text { the greatest integer } \leq 2 \sqrt{q} \text { prime to } q, \text { if } q \text { is not a perfect square; } \\
2 \sqrt{q}, \text { if } q \text { is a perfect square. }
\end{array}\right.
$$

Proof. Suppose that the lemma is false. Then there exists an integer $0<j<d$ such that $j<\frac{1}{2}(q+1+\epsilon(q))$ if $q \geq 4$ (respectively $j \leq \frac{1}{2} q+1$ if $q \in\{2,3\}$ ) and $\mu_{q}^{\mathrm{sym}}(j)>\frac{j}{d} \mu_{q}^{\mathrm{sym}}(d)$. Two cases can occur:

- either $j \leq \frac{q}{2}+1$ (in particular, this is the case if $q \in\{2,3\}$ ), and then we have $\mu_{q}^{\mathrm{sym}}(j)>\frac{j}{d} \mu_{q}^{\mathrm{sym}}(d) \geq \frac{j}{d}(2 d-1)>2 j-1$,

- or $\frac{q}{2}+1<j<\frac{1}{2}(q+1+\epsilon(q))$, so $\mu_{q}^{\mathrm{sym}}(d) \geq 2 d$ leads to $\mu_{q}^{\mathrm{sym}}(j)>$ $\frac{j}{d} \mu_{q}^{\mathrm{sym}}(d) \geq 2 j$,

so both cases contradict the results recalled in Section 1.3

Proposition 2.2. Let $q$ be a prime power and $d$ a positive integer such that any proper divisor $j$ of d satisfies $j<\frac{1}{2}(q+1+\epsilon(q))$ if $q \geq 4$ (with the same definition for $\epsilon$ as in Lemma 2.1) or $j \leq \frac{1}{2} q+1$ if $q \in\{2,3\}$. Let $F / \mathbb{F}_{q}$ be an algebraic function field of genus $g \geq 2$ with $N_{i}$ places of degree $i$ and let $l_{i}$ be integers such that $0 \leq l_{i} \leq N_{i}$, for all $i \mid d$. Suppose that:

(i) there exists a place of degree $n$ of $F / \mathbb{F}_{q}$, 
(ii) $\sum_{i \mid d} i\left(N_{i}+l_{i}\right) \geq 2 n+g+\alpha_{q}$, where $\alpha_{2}=5, \alpha_{3}=\alpha_{4}=\alpha_{5}=2$ and $\alpha_{q}=-1$ for $q>5$.

Then

$$
\mu_{q}(n) \leq \frac{2 \mu_{q}^{\mathrm{sym}}(d)}{d}\left(n+\frac{g}{2}\right)+\gamma_{q, d} \sum_{i \mid d} i l_{i}+\kappa_{q, d}
$$

where $\gamma_{q, d}:=\max _{i \mid d}\left(\frac{\mu_{q}(i, 2)}{i}\right)-\frac{2 \mu_{q}^{\mathrm{sym}}(d)}{d}$ and $\kappa_{q, d} \leq \frac{\mu_{q}^{\mathrm{sym}}(d)}{d}\left(\alpha_{q}+d-1\right)$.

Proof. We apply Theorem 1.3 with $n_{i, 1}=N_{i}-l_{i}$ and $n_{i, 2}=l_{i}$ for any $i \mid d$, and the others $n_{j, u}=0$. We choose $l=1$ and $m=n$ and we get

$$
\begin{aligned}
\mu_{q}(n) \leq & \sum_{i \mid d}\left(n_{i, 1} \mu_{q}(i)+n_{i, 2} \mu_{q}(i, 2)\right) \\
= & \sum_{i \mid d}\left(\left(N_{i}-l_{i}\right) \mu_{q}(i)+l_{i} \mu_{q}(i, 2)\right) \\
\leq & \sum_{i \mid d}\left(\left(N_{i}-l_{i}\right) \mu_{q}^{\mathrm{sym}}(i)+l_{i} \mu_{q}(i, 2)\right) \\
= & \sum_{i \mid d}\left(\left(N_{i}+l_{i}\right) \mu_{q}^{\mathrm{sym}}(i)+l_{i}\left(\mu_{q}(i, 2)-2 \mu_{q}^{\mathrm{sym}}(i)\right)\right) \\
= & \sum_{i \mid d}\left(i\left(N_{i}+l_{i}\right) \frac{\mu_{q}^{\mathrm{sym}}(i)}{i}+i l_{i}\left(\frac{\mu_{q}(i, 2)-2 \mu_{q}^{\mathrm{sym}}(i)}{i}\right)\right) \\
= & \frac{\mu_{q}^{\mathrm{sym}}(d)}{d} \sum_{i \mid d} i\left(N_{i}+l_{i}\right)+\sum_{i \mid d}\left(i\left(N_{i}+l_{i}\right)\left(\frac{\mu_{q}^{\mathrm{sym}}(i)}{i}-\frac{\mu_{q}^{\mathrm{sym}}(d)}{d}\right)\right. \\
& \left.+i l_{i}\left(\frac{\mu_{q}(i, 2)-2 \mu_{q}^{\mathrm{sym}}(i)}{i}\right)\right) \\
\leq & \frac{\mu_{q}^{\mathrm{sym}}(d)}{d} \sum_{i \mid d} i\left(N_{i}+l_{i}\right)+\sum_{i \mid d} i l_{i}\left(\frac{\mu_{q}(i, 2)-\mu_{q}^{\mathrm{sym}}(i)}{i}-\frac{\mu_{q}^{\mathrm{sym}}(d)}{d}\right) \\
& +\sum_{i \mid d} i N_{i}\left(\frac{\mu_{q}^{\mathrm{sym}}(i)}{i}-\frac{\mu_{q}^{\mathrm{sym}}(d)}{d}\right) .
\end{aligned}
$$

According to Lemma 2.1, we have $\frac{\mu_{q}^{\mathrm{sym}}(i)}{i}-\frac{\mu_{q}^{\mathrm{sym}}(d)}{d} \leq 0$, so

$$
\sum_{i \mid d} i N_{i}\left(\frac{\mu_{q}^{\mathrm{sym}}(i)}{i}-\frac{\mu_{q}^{\mathrm{sym}}(d)}{d}\right) \leq \sum_{i \mid d} i l_{i}\left(\frac{\mu_{q}^{\mathrm{sym}}(i)}{i}-\frac{\mu_{q}^{\mathrm{sym}}(d)}{d}\right)
$$

since $0 \leq l_{i} \leq N_{i}$ for any $i \mid d$. Moreover, w.l.o.g., we can suppose from (ii) that $\sum_{i \mid d} i\left(N_{i}+l_{i}\right)=2 n+g+\alpha_{q}+k_{d}$, with $k_{d} \in\{0, \ldots, d-1\}$. We obtain

$$
\mu_{q}(n) \leq \frac{\mu_{q}^{\mathrm{sym}}(d)}{d}\left(2 n+g+\alpha_{q}+k_{d}\right)+\sum_{i \mid d} i l_{i}\left(\frac{\mu_{q}(i, 2)}{i}-\frac{2 \mu_{q}^{\mathrm{sym}}(d)}{d}\right)
$$

which gives the result. 
The two following corollaries are straightforward and give explicit values for bound (3) obtained from the preceding proposition applied for the special cases where $d=1,2$ or 4 .

Corollary 2.3. Let $q \geq 3$ be a prime power and let $F / \mathbb{F}_{q}$ be an algebraic function field of genus $g \geq 2$ with $N_{i}$ places of degree $i$ and let $l_{i}$ be integers such that $0 \leq l_{i} \leq N_{i}$. If

(i) there exists a place of degree $n$ of $F / \mathbb{F}_{q}$,

(ii) $N_{1}+l_{1}+2\left(N_{2}+l_{2}\right) \geq 2 n+g+\alpha_{q}$, where $\alpha_{3}=\alpha_{4}=\alpha_{5}=2$ and $\alpha_{q}=-1$, for $q>5$,

then

and for $q>5$,

$$
\begin{gathered}
\mu_{3}(n) \leq 3 n+\frac{3}{2} g+\frac{3}{2}\left(l_{1}+2 l_{2}\right)+\frac{9}{2}, \\
\mu_{4}(n) \text { and } \mu_{5}(n) \leq 3 n+\frac{3}{2} g+l_{1}+2 l_{2}+\frac{9}{2},
\end{gathered}
$$

$$
\mu_{q}(n) \leq \begin{cases}2 n+g+l_{1}-1 & \text { if } N_{2}=l_{2}=0 \\ 3 n+\frac{3}{2} g+\frac{1}{2}\left(l_{1}+2 l_{2}\right) & \text { else. }\end{cases}
$$

Proof. To apply Proposition 2.2 , let us recall that $\mu_{q}^{\mathrm{sym}}(2)=3$ and $\mu_{q}(1,2) \leq 3$ for any prime power $q$. Moreover, according to [18, Example 4.4], one knows that $\mu_{3}(2,2) \leq 9, \mu_{q}(2,2) \leq 8$ for $q=4$ or 5 and $\mu_{q}(2,2) \leq 7$ for $q>5$. Hence, we can deduce that $\gamma_{3,2} \leq \frac{9}{2}-3=\frac{3}{2}, \gamma_{q, 2} \leq \frac{8}{2}-3=1$ for $q=4$ or 5 , and $\gamma_{q, 2} \leq \frac{7}{2}-3=\frac{1}{2}$ and $\gamma_{q, 1} \leq 1$ for $q>5$. (In case $q>5, N_{2}=l_{2}=0$, we take $d=1$ in Prop. 2.2).

Corollary 2.4. Let $F / \mathbb{F}_{2}$ be an algebraic function field of genus $g \geq 2$ with $N_{i}$ places of degree $i$ and let $l_{i}$ be integers such that $0 \leq l_{i} \leq N_{i}$. If

(i) there exists a place of degree $n$ of $F / \mathbb{F}_{2}$,

(ii) $\sum_{i \mid 4} i\left(N_{i}+l_{i}\right) \geq 2 n+g+5$,

then

$$
\mu_{2}(n) \leq \frac{9}{2}\left(n+\frac{g}{2}\right)+\frac{3}{2} \sum_{i \mid 4} i l_{i}+18 .
$$

Proof. We recall from [14, Example 6.1] that $\mu_{2}^{\text {sym }}(4)=9$ and from [18, Example 4.4, Lemma 4.6] that $\mu_{2}(2,2) \leq 9$ and $\mu_{2}(4,2) \leq 24$, which gives $\gamma_{2,4} \leq \frac{24}{4}-\frac{2 \cdot 9}{4}=\frac{3}{2}$.

2.1. General method to obtain uniform bounds for $\mu_{q}(n)$. We consider a tower $\mathcal{F}$ of function fields $F_{i} / \mathbb{F}_{q}$ of genus $g\left(F_{i}\right)$ with $B_{\ell}\left(F_{i}\right)$ places of degree $\ell$. Let $d$ be an integer such that any proper divisor $j$ of $d$ satisfies $j<\frac{1}{2}(q+1+\epsilon(q))$ if $q \geq 4$ (with the same definition for $\epsilon$ as in Lemma 2.1), or $j \leq \frac{1}{2} q+1$ if $q \in\{2,3\}$.

Suppose there exists an integer $N$ such that, for all $n \geq N$, there is an integer $k(n)$ for which:
(A) $\sum_{j \mid d} j B_{j}\left(F_{k(n)+1}\right) \geq 2 n+g\left(F_{k(n)+1}\right)+\alpha_{q}$ and $B_{n}\left(F_{k(n)+1}\right)>0$,
(B) $\sum_{j \mid d} j B_{j}\left(F_{k(n)}\right)<2 n+g\left(F_{k(n)}\right)+\alpha_{q}$ but $B_{n}\left(F_{k(n)}\right)>0$,
(C) $g\left(F_{k(n)}\right) \geq 2\left(\right.$ so $\left.g\left(F_{k(n)+1}\right) \geq 2\right)$,
(D) $\Delta g_{k(n)}:=g\left(F_{k(n)+1}\right)-g\left(F_{k(n)}\right) \geq \lambda D_{k(n)}$ with $\lambda:=\frac{d \gamma_{q, d}}{\mu_{q}^{\text {sym }}(d)}$,
(E) $\sum_{j \mid d} j B_{j}\left(F_{k(n)}\right) \geq D_{k(n)}$, 
where $\alpha_{q}$ is as in Proposition 2.2 and $D_{k(n)}$ is chosen to satisfy (D) and (E), and is fixed for the tower $\mathcal{F}$.

We also set

$$
n_{0}^{l}:=\sup \left\{m \in \mathbb{N} \mid \sum_{j \mid d} j B_{j}\left(F_{l}\right) \geq 2 m+g\left(F_{l}\right)+\alpha_{q}\right\} .
$$

Note that for the integer $n_{0}^{k(n)}$, the following holds:

$$
\sum_{j \mid d} j B_{j}\left(F_{k(n)}\right)+2\left(n-n_{0}^{k(n)}\right) \geq 2 n+g\left(F_{k(n)}\right)+\alpha_{q} .
$$

Now, fix an integer $n \geq N$ and let $k:=k(n)$ satisfying hypotheses (A) to (E).

To multiply in $\mathbb{F}_{q^{n}}$, one has the following alternative:

(a) apply the algorithm on the step $F_{k+1}$, with $B_{j}\left(F_{k+1}\right)$ places of degree $j$ for any $j \mid d$, all of them used with multiplicity 1 ; this is possible according to (A) and (C). In this case, Proposition 2.2 gives the following bound for $\mu_{q}(n)$ :

$$
\mu_{q}(n) \leq \frac{2 \mu_{q}^{\mathrm{sym}}(d)}{d}\left(n+\frac{g\left(F_{k+1}\right)}{2}\right)+\frac{\mu_{q}^{\mathrm{sym}}(d)}{d}\left(\alpha_{q}+d-1\right) ;
$$

(b) apply the algorithm on the step $F_{k}$, with $B_{j}\left(F_{k}\right)$ places of degree $j$ of which $l_{j}$ used with multiplicity 2 and the remaining with multiplicity 1 , for any $j \mid d$, where the integers $l_{j} \leq B_{j}\left(F_{k}\right)$ satisfy $\sum_{j \mid d} l_{j} \geq 2\left(n-n_{0}^{k}\right)$; for such integers $l_{j}$, we can apply Proposition 2.2 according to (B) and (4). In particular, if $2\left(n-n_{0}^{k}\right)+d-1 \leq \sum_{j \mid d} j B_{j}\left(F_{k}\right)$, then we can choose the integers $l_{j}$ such that $\sum_{j \mid d} j l_{j}=2\left(n-n_{0}^{k}\right)+\epsilon$ for some $\epsilon \in\{0, \ldots, d-1\}$, and this is a suitable choice. In this case, Proposition 2.2 gives

$$
\mu_{q}(n) \leq \frac{2 \mu_{q}^{\mathrm{sym}}(d)}{d}\left(n+\frac{g\left(F_{k}\right)}{2}\right)+\gamma_{q, d} \sum_{i \mid d} i l_{i}+\frac{\mu_{q}^{\mathrm{sym}}(d)}{d}\left(\alpha_{q}+d-1\right) .
$$

Note that we can rewrite (5) as

$$
\mu_{q}(n) \leq \frac{2 \mu_{q}^{\mathrm{sym}}(d)}{d}\left(n+\frac{g\left(F_{k}\right)}{2}\right)+\frac{\mu_{q}^{\mathrm{sym}}(d)}{d} \Delta g_{k}+\frac{\mu_{q}^{\mathrm{sym}}(d)}{d}\left(\alpha_{q}+d-1\right),
$$

which makes clear that if $\gamma_{q, d} \sum_{i \mid d} i l_{i}<\frac{\mu_{q}^{\mathrm{sym}}(d)}{d} \Delta g_{k}$, then case (b) gives a better bound than case (a) .

So if $2\left(n-n_{0}^{k}\right)+d-1<D_{k}$, then we can proceed as in case (b) since according to hypothesis (E) we can choose $\epsilon \in\{0, \ldots, d-1\}$ and $l_{j}$ for $j \mid d$ such that $\sum_{j \mid d} j l_{j}=2\left(n-n_{0}^{k}\right)+\epsilon$. Moreover, we have

$$
\frac{d \gamma_{q, d}}{\mu_{q}^{\text {sym }}(d)}\left(2\left(n-n_{0}^{k}\right)+d-1\right)<\Delta g_{k}
$$

from hypothesis (D) , so $\gamma_{q, d}\left(2\left(n-n_{0}^{k}\right)+\epsilon\right)<\frac{\mu_{q}^{\mathrm{sym}}(d)}{d} \Delta g_{k}$ which means that the bound obtained from case (b) is sharper. 
For $x \in \mathbb{R}^{+}, \quad x \geq N$, such that $\sum_{j \mid d} j B_{j}\left(F_{k+1}\right) \geq 2[x]+g\left(F_{k+1}\right)+\alpha_{q}$ and $\sum_{j \mid d} j B_{j}\left(F_{k}\right)<2[x]+g\left(F_{k}\right)+\alpha_{q}$, we define the function $\Phi_{k}(x)$ as follows:

$\Phi_{k}(x)=\left\{\begin{array}{c}\frac{2 \mu_{q}^{\mathrm{sym}}(d)}{d}\left(x+\frac{g\left(F_{k}\right)}{2}\right)+\gamma_{q, d}\left(2\left(x-n_{0}^{k}\right)+d-1\right)+\frac{\mu_{q}^{\mathrm{sym}}(d)}{d}\left(\alpha_{q}+d-1\right), \\ \text { if } 2\left(x-n_{0}^{k}\right)+d-1<D_{k} ; \\ \frac{2 \mu_{q}^{\mathrm{sym}}(d)}{d}\left(x+\frac{g\left(F_{k+1}\right)}{2}\right)+\frac{\mu_{q}^{\mathrm{sym}}(d)}{d}\left(\alpha_{q}+d-1\right), \text { else. }\end{array}\right.$

that is to say:

$$
\Phi_{k}(x)=\left\{\begin{array}{r}
\left(\frac{2 \mu_{q}^{\mathrm{sym}}(d)}{d}+2 \gamma_{q, d}\right)\left(x-n_{0}^{k}\right)+\frac{\mu_{q}^{\mathrm{sym}}(d)}{d}\left(2 n_{0}^{k}+g\left(F_{k}\right)+\alpha_{q}+d-1\right), \\
\text { if } 2\left(x-n_{0}^{k}\right)+d-1<D_{k} . \\
\frac{2 \mu_{q}^{\mathrm{sym}}(d)}{d}\left(x-n_{0}^{k}\right)+\frac{\mu_{q}^{\mathrm{sym}}(d)}{d}\left(2 n_{0}^{k}+g\left(F_{k+1}\right)+\alpha_{q}+d-1\right), \text { else. }
\end{array}\right.
$$

We define the function $\Phi$ for all $x \geq N$ as the minimum of the functions $\Phi_{i}$ for which $x$ is in the domain of $\Phi_{i}$. This function is piecewise linear with two kinds of pieces: those which have slope $\frac{2 \mu_{q}^{\mathrm{sym}}(d)}{d}$ and those which have slope $\frac{2 \mu_{q}^{\mathrm{sym}}(d)}{d}+2 \gamma_{q, d}$. Moreover, the graph of the function $\Phi$ lies below any straight line that lies above all the points $\left(n_{0}^{i}+\frac{1}{2}\left(D_{i}-d+1\right), \Phi\left(n_{0}^{i}+\frac{1}{2}\left(D_{i}-d+1\right)\right)\right)$, since these are the vertices of the graph. Let $X:=n_{0}^{i}+\frac{1}{2}\left(D_{i}-d+1\right)$, then

$$
\begin{aligned}
\Phi(X) & =\frac{2 \mu_{q}^{\mathrm{sym}}(d)}{d}\left(X+\frac{g\left(F_{i+1}\right)}{2}\right)+\frac{\mu_{q}^{\mathrm{sym}}(d)}{d}\left(\alpha_{q}+d-1\right) \\
& =\frac{2 \mu_{q}^{\mathrm{sym}}(d)}{d}\left(1+\frac{g\left(F_{i+1}\right)}{2 X}\right) X+\frac{\mu_{q}^{\mathrm{sym}}(d)}{d}\left(\alpha_{q}+d-1\right) .
\end{aligned}
$$

If we can give a bound for $\Phi(X)$ which is independent of $i$, then it will provide a bound for $\mu_{q}(n)$ for all $n \geq N$, since $\mu_{q}(n) \leq \Phi(n)$.

\section{Good SEQUENCES OF FUnCTION FIELDS}

3.1. Garcia-Stichtenoth tower of Artin-Schreier algebraic function field extensions. We present now a modified Garcia-Stichtenoth tower (cf. [16], 2], [9]) having good properties. Let us consider a finite field $\mathbb{F}_{q^{2}}$ with $q=p^{r} \geq 4$ and $r$ an integer. We consider the Garcia-Stichtenoth elementary abelian tower $T_{1}$ over $\mathbb{F}_{q^{2}}$ constructed in [16] and defined by the sequence $\left(F_{1}, F_{2}, F_{3}, \ldots\right)$ where

$$
F_{k+1}:=F_{k}\left(z_{k+1}\right)
$$

and $z_{k+1}$ satisfies the equation

$$
z_{k+1}^{q}+z_{k+1}=x_{k}^{q+1}
$$

with

$$
x_{k}:=z_{k} / x_{k-1} \text { in } F_{k}(\text { for } k \geq 2) .
$$

Moreover, $F_{1}:=\mathbb{F}_{q^{2}}\left(x_{1}\right)$ is the rational function field over $\mathbb{F}_{q^{2}}$ and $F_{2}$ the Hermitian function field over $\mathbb{F}_{q^{2}}$. Let us denote by $g_{k}$ the genus of $F_{k}$, we recall the following formulae:

$$
g_{k}=\left\{\begin{array}{lll}
q^{k}+q^{k-1}-q^{\frac{k+1}{2}}-2 q^{\frac{k-1}{2}}+1 & \text { if } k \equiv 1 \quad \bmod 2 \\
q^{k}+q^{k-1}-\frac{1}{2} q^{\frac{k}{2}+1}-\frac{3}{2} q^{\frac{k}{2}}-q^{\frac{k}{2}-1}+1 & \text { if } k \equiv 0 \quad \bmod 2
\end{array}\right.
$$

Let us consider the completed Garcia-Stichtenoth tower

$$
T_{2}=F_{1,0} \subseteq F_{1,1} \subseteq \cdots \subseteq F_{1, r}=F_{2,0} \subseteq F_{2,1} \subseteq \cdots \subseteq F_{2, r} \subseteq \cdots
$$


considered in [2] such that $F_{k} \subseteq F_{k, s} \subseteq F_{k+1}$ for any integer $s \in\{0, \ldots, r\}$, with $F_{k, 0}=F_{k}$ and $F_{k, r}=F_{k+1}$. Recall that each extension $F_{k, s} / F_{k}$ is Galois of degree $p^{s}$ with full constant field $\mathbb{F}_{q^{2}}$. Now, we consider the tower studied in 9]

$$
T_{3}=G_{1,0} \subseteq G_{1,1} \subseteq \cdots \subseteq G_{1, r}=G_{2,0} \subseteq G_{2,1} \subseteq \cdots \subseteq G_{2, r} \subseteq \cdots
$$

defined over the constant field $\mathbb{F}_{q}$ and related to the tower $T_{2}$ by

$$
F_{k, s}=\mathbb{F}_{q^{2}} G_{k, s} \text { for all } k \text { and } s,
$$

namely $F_{k, s} / \mathbb{F}_{q^{2}}$ is the constant field extension of $G_{k, s} / \mathbb{F}_{q}$. Note that the tower $T_{3}$ is well defined by [9] and [7]. Moreover, we have the following result:

Proposition 3.1. Let $q=p^{r} \geq 4$ be a prime power. For all integers $k \geq 1$ and $s \in\{0, \ldots, r\}$, there exists a step $F_{k, s} / \mathbb{F}_{q^{2}}$ (respectively $G_{k, s} / \mathbb{F}_{q}$ ) with genus $g_{k, s}$ and $N_{k, s}$ places of degree one in $F_{k, s} / \mathbb{F}_{q^{2}}$ (resp. $N_{k, s}:=B_{1}\left(G_{k, s} / \mathbb{F}_{q}\right)+2 B_{2}\left(G_{k, s} / \mathbb{F}_{q}\right)$ where $B_{i}\left(G_{k, s} / \mathbb{F}_{q}\right)$ denote the number of places of degree $i$ in $\left.G_{k, s} / \mathbb{F}_{q}\right)$ such that:

(1) $F_{k} \subseteq F_{k, s} \subseteq F_{k+1}$, where we set $F_{k, 0}:=F_{k}$ and $F_{k, r}:=F_{k+1}$,

(resp. $G_{k} \subseteq G_{k, s} \subseteq G_{k+1}$, with $G_{k, 0}:=G_{k}$ and $G_{k, r}:=G_{k+1}$ ),

(2) $\left(g_{k}-1\right) p^{s}+1 \leq g_{k, s} \leq \frac{g_{k+1}}{p^{r-s}}+1$,

(3) $N_{k, s} \geq\left(q^{2}-1\right) q^{k-1} p^{s}$.

Now, we are interested to search the descent of the definition field of the tower $T_{2} / \mathbb{F}_{q^{2}}$ from $\mathbb{F}_{q^{2}}$ to $\mathbb{F}_{p}$ if it is possible. In fact, one cannot establish a general result but one can prove that it is possible in the case of characteristic 2 which is given by the following result obtained in [10.

Proposition 3.2. Let $p=2$. If $q=p^{2}$, the descent of the definition field of the tower $T_{2} / \mathbb{F}_{q^{2}}$ from $\mathbb{F}_{q^{2}}$ to $\mathbb{F}_{p}$ is possible. More precisely, there exists a tower $T_{4} / \mathbb{F}_{p}$ defined over $\mathbb{F}_{p}$ given by a sequence:

$$
T_{4} / \mathbb{F}_{p}=H_{1,0} \subseteq H_{1,1} \subseteq H_{1,2}=H_{2,0} \subseteq H_{2,1} \subseteq H_{2,2}=H_{3,0} \subseteq \cdots
$$

defined over the constant field $\mathbb{F}_{p}$ and related to the towers $T_{1} / \mathbb{F}_{q^{2}}$ and $T_{2} / \mathbb{F}_{q}$ by

$$
\begin{aligned}
& F_{k, s}=\mathbb{F}_{q^{2}} H_{k, s} \text { for all } k \text { and } s=0,1,2, \\
& G_{k, s}=\mathbb{F}_{q} H_{k, s} \text { for all } k \text { and } s=0,1,2,
\end{aligned}
$$

namely $F_{k, s} / \mathbb{F}_{q^{2}}$ is the constant field extension of $G_{k, s} / \mathbb{F}_{q}$ and $H_{k, s} / \mathbb{F}_{p}$ and $G_{k, s} / \mathbb{F}_{q}$ is the constant field extension of $H_{k, s} / \mathbb{F}_{p}$.

Moreover, from [10], the following properties holds for this tower $T_{3} / \mathbb{F}_{p}$ :

Proposition 3.3. Let $q=p^{2}=4$. For any integers $k \geq 1$ and $s \in\{0,1,2\}$, the algebraic function field $H_{k, s} / \mathbb{F}_{p}$ in the tower $T_{3} / \mathbb{F}_{p}$ with genus $g_{k, s}:=g\left(H_{k, s} / \mathbb{F}_{p}\right)$ and $B_{i}\left(H_{k, s} / \mathbb{F}_{p}\right)$ places of degree $i$, is such that:

(1) $H_{k} / \mathbb{F}_{p} \subseteq H_{k, s} / \mathbb{F}_{p} \subseteq H_{k+1} / \mathbb{F}_{p}$ with $H_{k, 0}=H_{k}$ and $H_{k, 2}=H_{k+1}$,

(2) $g_{k, s} \leq \frac{g_{k+1}}{p^{2-s}}+1$ with $g_{k+1} \leq q^{k+1}+q^{k}$,

(3) $B_{1}\left(H_{k, s} / \mathbb{F}_{p}\right)+2 B_{2}\left(H_{k, s} / \mathbb{F}_{p}\right)+4 B_{4}\left(H_{k, s} / \mathbb{F}_{p}\right) \geq\left(q^{2}-1\right) q^{k-1} p^{s}$.

3.2. Garcia-Stichtenoth tower of Kummer function field extensions. In this section we present another Garcia-Stichtenoth tower (cf. 4]) having good properties. Let $\mathbb{F}_{q}$ be a finite field of characteristic $p \geq 3$. Let us consider the tower $T$ over $\mathbb{F}_{q}$ which is defined recursively by the following equation, studied in [17]:

$$
y^{2}=\frac{x^{2}+1}{2 x} \text {. }
$$


The tower $T / \mathbb{F}_{q}$ is represented by the sequence of function fields $\left(L_{0}, L_{1}, L_{2}, \ldots\right)$ where $L_{n}=\mathbb{F}_{q}\left(x_{0}, x_{1}, \ldots, x_{n}\right)$ and $x_{i+1}^{2}=\left(x_{i}^{2}+1\right) / 2 x_{i}$ holds for each $i \geq 0$. Note that $L_{0}$ is the rational function field. For any prime number $p \geq 3$, the tower $T / \mathbb{F}_{p^{2}}$ is asymptotically optimal over the field $\mathbb{F}_{p^{2}}$, i.e., $T / \mathbb{F}_{p^{2}}$ reaches the Drinfeld-Vlădut bound. Moreover, for any integer $k, L_{k} / \mathbb{F}_{p^{2}}$ is the constant field extension of $L_{k} / \mathbb{F}_{p}$. by

From [4, we know that the genus $g\left(L_{k}\right)$ of the steps $L_{k} / \mathbb{F}_{p^{2}}$ and $L_{k} / \mathbb{F}_{p}$ is given

$$
g\left(L_{k}\right)= \begin{cases}2^{k+1}-3 \cdot 2^{\frac{k}{2}}+1 & \text { if } k \equiv 0 \quad \bmod 2 \\ 2^{k+1}-2 \cdot 2^{\frac{k+1}{2}}+1 & \text { if } k \equiv 1 \quad \bmod 2\end{cases}
$$

and that the following bounds hold for the number of rational places in $L_{k}$ over $\mathbb{F}_{p^{2}}$ and for the number of places of degrees one and two over $\mathbb{F}_{p}$ :

$$
B_{1}\left(L_{k} / \mathbb{F}_{p^{2}}\right) \geq 2^{k+1}(p-1)
$$

and

$$
B_{1}\left(L_{k} / \mathbb{F}_{p}\right)+2 B_{2}\left(L_{k} / \mathbb{F}_{p}\right) \geq 2^{k+1}(p-1) .
$$

3.3. Some preliminary results. Here we establish some technical results about the genus and number of places of each step of the towers $T_{2} / \mathbb{F}_{q^{2}}, T_{3} / \mathbb{F}_{q}, T_{4} / \mathbb{F}_{2}$, $T / \mathbb{F}_{p^{2}}$ and $T / \mathbb{F}_{p}$ defined in Sections 3.1 and 3.2. These results will allow us to determine a suitable step of the tower to apply the algorithm on.

3.3.1. About the Garcia-Stichtenoth tower of Artin-Schreier extensions. In this section, $q=p^{r}$ is a power of the prime $p$. We denote by $g_{k, s}$ the genus of the corresponding steps of the towers $T_{2} / \mathbb{F}_{q^{2}}, T_{3} / \mathbb{F}_{q}$ and $T_{4} / \mathbb{F}_{2} ;$ recall that $g_{k}=g_{k, 0}=g_{k-1, r}$. We also set

$$
\Delta g_{k, s}:=g_{k, s+1}-g_{k, s} .
$$

Lemma 3.4. Let $q \geq 4$. We have the following bounds for the genus of each step of the towers $T_{2} / \mathbb{F}_{q^{2}}, T_{3} / \mathbb{F}_{q}$ and $T_{4} / \mathbb{F}_{2}$ (we set $q=4$ and $p=r=2$ in the special case of this tower):

i) $g_{k}>q^{k}$ for all $k \geq 4$;

moreover, for the tower $T_{4} / \mathbb{F}_{2}$, one has $g_{k}>p q^{k-1}$ for all $k \geq 3$,

ii) $g_{k} \leq q^{k-1}(q+1)-\sqrt{q} q^{\frac{k}{2}}$,

iii) $g_{k, s} \leq q^{k-1}(q+1) p^{s}$ for all $k \geq 0$ and $s \in\{0, \ldots, r\}$,

iv) $g_{k, s} \leq \frac{q^{k}(q+1)-q^{\frac{k}{2}}(q-1)}{p^{r-s}}$ for all $k \geq 2$ and $s \in\{0, \ldots, r\}$.

Proof. i) According to formula (7), we know that if $k \equiv 1 \bmod 2$, then

$$
g_{k}=q^{k}+q^{k-1}-q^{\frac{k+1}{2}}-2 q^{\frac{k-1}{2}}+1=q^{k}+q^{\frac{k-1}{2}}\left(q^{\frac{k-1}{2}}-q-2\right)+1 .
$$

Since $q>3$ and $k \geq 4$, we have $q^{\frac{k-1}{2}}-q-2>0$, thus $g_{k}>q^{k}$.

Else if $k \equiv 0 \bmod 2$, then

$$
g_{k}=q^{k}+q^{k-1}-\frac{1}{2} q^{\frac{k}{2}+1}-\frac{3}{2} q^{\frac{k}{2}}-q^{\frac{k}{2}-1}+1=q^{k}+q^{\frac{k}{2}-1}\left(q^{\frac{k}{2}}-\frac{1}{2} q^{2}-\frac{3}{2} q-1\right)+1 .
$$

Since $q>3$ and $k \geq 4$, we have $q^{\frac{k}{2}}-\frac{1}{2} q^{2}-\frac{3}{2} q-1>0$, thus $g_{k}>q^{k}$.

Hence, the second bound for the tower $T_{4} / \mathbb{F}_{2}$ is already proved for $k \geq 4$, and for $k=3$, one has $g_{3}-p q^{2}=q^{3}-2 q+1-p q^{2}=25$ so this bound holds also for $k=3$. 
ii) It follows from formula (7) since for all $k \geq 1$ we have $2 q^{\frac{k-1}{2}} \geq 1$ which works out for odd $k$ cases and $\frac{3}{2} q^{\frac{k}{2}}+q^{\frac{k}{2}-1} \geq 1$ which works out for even $k$ cases, since $\frac{1}{2} q \geq \sqrt{q}$.

iii) If $s=r$, then according to formula (7), we have

$$
g_{k, s}=g_{k+1} \leq q^{k+1}+q^{k}=q^{k-1}(q+1) p^{s} .
$$

Else, $s<r$ and Proposition 3.1 says that $g_{k, s} \leq \frac{g_{k+1}}{p^{r-s}}+1$. Moreover, since $q^{\frac{k+2}{2}} \geq q$ and $\frac{1}{2} q^{\frac{k+1}{2}+1} \geq q$, we obtain $g_{k+1} \leq q^{k+1}+q^{k}-q+1$ from formula (7). Thus, we get

$$
\begin{aligned}
g_{k, s} & \leq \frac{q^{k+1}+q^{k}-q+1}{p^{r-s}}+1 \\
& =q^{k-1}(q+1) p^{s}-p^{s}+p^{s-r}+1 \\
& \leq q^{k-1}(q+1) p^{s}+p^{s-r} \\
& \leq q^{k-1}(q+1) p^{s} \text { since } 0 \leq p^{s-r}<1 \text { and } g_{k, s} \in \mathbb{N} .
\end{aligned}
$$

iv) It follows from ii) since Proposition 3.1 gives $g_{k, s} \leq \frac{g_{k+1}}{p^{r-s}}+1$, so

$$
g_{k, s} \leq \frac{q^{k}(q+1)-\sqrt{q} q^{\frac{k+1}{2}}}{p^{r-s}}+1,
$$

which gives the result since $p^{r-s} \leq q^{\frac{k}{2}}$ for all $k \geq 2$.

Now we set $N_{k, s}:=B_{1}\left(F_{k, s} / \mathbb{F}_{q^{2}}\right)=B_{1}\left(G_{k, s} / \mathbb{F}_{q}\right)+2 B_{2}\left(G_{k, s} / \mathbb{F}_{q}\right)$.

Lemma 3.5. Let $D_{k, s}:=(p-1) p^{s} q^{k}$. For any $k \geq 1$ and $s \in\{0, \ldots, r-1\}$, one has:

i) $\Delta g_{k, s} \geq D_{k, s}$ if $k \geq 4$,

ii) $N_{k, s} \geq D_{k, s}$.

Proof. i) From the Hurwitz Genus Formula, one has $g_{k, s+1}-1 \geq p\left(g_{k, s}-1\right)$, so

$$
g_{k, s+1}-g_{k, s} \geq(p-1)\left(g_{k, s}-1\right) .
$$

Applying $s$ more times the Hurwitz Genus Formula, we get $g_{k, s+1}-g_{k, s} \geq$ $(p-1) p^{s}\left(g_{k}-1\right)$. Thus we have $g_{k, s+1}-g_{k, s} \geq(p-1) p^{s} q^{k}$, from Lemma 3.4 i) since $q>3$ and $k \geq 4$.

ii) According to Proposition 3.1 one has

$$
\begin{aligned}
N_{k, s} & \geq\left(q^{2}-1\right) q^{k-1} p^{s} \\
& =(q+1)(q-1) q^{k-1} p^{s} \\
& \geq(q-1) q^{k} p^{s} \\
& \geq(p-1) q^{k} p^{s} .
\end{aligned}
$$

Lemma 3.6. For all $k \geq 1$ and $s \in\{0, \ldots, r\}$, one has

$$
\sup \left\{n \in \mathbb{N} \mid N_{k, s} \geq 2 n+g_{k, s}-1\right\} \geq \frac{1}{2}(q+1) q^{k-1} p^{s}(q-2)+\frac{1}{2} .
$$

Proof. From Proposition 3.1 and Lemma 3.4 iii), we get

$$
\begin{aligned}
N_{k, s}-g_{k, s}+1 & \geq\left(q^{2}-1\right) q^{k-1} p^{s}-q^{k-1}(q+1) p^{s}+1 \\
& =(q+1) q^{k-1} p^{s}((q-1)-1)+1 .
\end{aligned}
$$


Now we recall similar technical results about genus and number of places of each step of the tower $T_{4} / \mathbb{F}_{2}$ defined in Section 3.1. In order to simplify the presentation, we still use the variables $p$ and $q$.

Lemma 3.7. Let $q=p^{2}=4$. For all $k \geq 1$ and $s \in\{0,1\}$, we set $D_{k, s}:=\frac{3}{2} p^{s+1} q^{k-1}$. Then we have

i) $\Delta g_{k, s} \geq \lambda D_{k, s}$, with $\lambda:=\frac{4 \gamma_{2,4}}{\mu_{2}^{\text {sym }}(4)} \leq \frac{3}{2}$ (see Section 2.1),

ii) $B_{1}\left(H_{k, s} / \mathbb{F}_{p}\right)+2 B_{2}\left(H_{k, s} / \mathbb{F}_{p}\right)+4 B_{4}\left(H_{k, s} / \mathbb{F}_{p}\right) \geq D_{k, s}$.

Proof. i) We apply the Genus Hurwitz Formula as in the proof of Lemma 3.5 to obtain $g_{k, s+1}-g_{k, s} \geq(p-1) p^{s}\left(g_{k}-1\right)$, so we get $\Delta g_{k, s} \geq(p-1) p^{s+1} q^{k-1}$ from Lemma 3.4 i) for $k \geq 3$, which gives the results. For $k=1$ and 2 , we check that the result is still valid since $g_{1}=0, g_{1,1}=2, g_{2}=6, g_{2,1}=23$ and $g_{3}=57$.

ii) It is obvious since $q^{2}-1>\frac{3}{2} p$ and since from Proposition 3.3 we have

$$
B_{1}\left(H_{k, s} / \mathbb{F}_{2}\right)+2 B_{2}\left(H_{k, s} / \mathbb{F}_{2}\right)+4 B_{4}\left(H_{k, s} / \mathbb{F}_{2}\right) \geq\left(q^{2}-1\right) q^{k-1} p^{s} .
$$

Lemma 3.8. Let $q=p^{2}=4$. For all $k \geq 1$ and $s \in\{0,1,2\}$, we have

$$
\sup \left\{n \in \mathbb{N} \mid \sum_{i=1,2,4} i B_{i}\left(H_{k, s} / \mathbb{F}_{2}\right) \geq 2 n+g_{k, s}+5\right\} \geq 5 p^{s} q^{k-1}-\frac{5}{2} \text {. }
$$

Proof. From Proposition 3.3 and Lemma 3.4 iii), we get

$$
\begin{aligned}
\sum_{i=1,2,4} i B_{i}\left(H_{k, s} / \mathbb{F}_{2}\right)-g_{k, s}-5 & \geq\left(q^{2}-1\right) q^{k-1} p^{s}-q^{k-1}(q+1) p^{s}-5 \\
& =p^{s} q^{k-1}(q+1)(q-2)-5
\end{aligned}
$$

thus we get the result since $q=4$.

3.3.2. About the Garcia-Stichtenoth tower of Kummer extensions. In this section, $p$ is an odd prime. We denote by $g_{k}$ the genus of the step $L_{k}$ and we fix

$$
N_{k}:=B_{1}\left(L_{k} / \mathbb{F}_{p^{2}}\right)=B_{1}\left(L_{k} / \mathbb{F}_{p}\right)+2 B_{2}\left(L_{k} / \mathbb{F}_{p}\right)
$$

and

$$
\Delta g_{k}:=g_{k+1}-g_{k} \text {. }
$$

The following lemma is straightforward according to formulae (8):

Lemma 3.9. These two bounds hold for the genus of each step of the towers $T / \mathbb{F}_{p^{2}}$ and $T / \mathbb{F}_{p}$ :

i) $g_{k} \leq 2^{k+1}-2 \cdot 2^{\frac{k+1}{2}}+1$,

ii) $g_{k} \leq 2^{k+1}$.

Lemma 3.10. For all $k \geq 0$, one has $N_{k} \geq \Delta g_{k} \geq 2^{k+1}-2^{\frac{k+1}{2}}$.

Proof. If $k$ is even, then $\Delta g_{k}=2^{k+1}-2^{\frac{k}{2}}$, else $\Delta g_{k}=2^{k+1}-2^{\frac{k+1}{2}}$, so the second equality holds trivially. Moreover, since $p \geq 3$, the first one follows from bounds (9) and (10) which gives $N_{k} \geq 2^{k+2}$. 
Lemma 3.11. Let $L_{k}$ be a step of one of the towers $T / \mathbb{F}_{p^{2}}$ or $T / \mathbb{F}_{p}$. One has:

$$
\sup \left\{n \in \mathbb{N} \mid N_{k} \geq 2 n+g_{k}-1\right\} \geq 2^{k}(p-2)+2^{\frac{k+1}{2}} \text {, if } p>5
$$

and

$$
\sup \left\{n \in \mathbb{N} \mid N_{k} \geq 2 n+g_{k}+2\right\} \geq 2^{k}(p-2)+2^{\frac{k+1}{2}}-1 \text {, if } p=5 \text { or } 3 .
$$

Proof. From bounds (9) and (10) for $N_{k}$ and Lemma 3.9 i), we get

$$
\begin{aligned}
N_{k}-g_{k}+1 & \geq 2^{k+1}(p-1)-\left(2^{k+1}-2 \cdot 2^{\frac{k+1}{2}}+1\right)+1 \\
& =2^{k+1}(p-2)+2 \cdot 2^{\frac{k+1}{2}} .
\end{aligned}
$$

Similarly, we get

$$
\begin{aligned}
N_{k}-g_{k}-2 & \geq 2^{k+1}(p-1)-\left(2^{k+1}-2 \cdot 2^{\frac{k+1}{2}}+1\right)-2 \\
& =2^{k+1}(p-2)+2 \cdot 2^{\frac{k+1}{2}}-3,
\end{aligned}
$$

which gives the result for $p=5$ or 3 .

3.4. Existence of a good step in each tower. The following lemmas prove the existence of a "good" step of the towers defined in Sections 3.1 and 3.2, that is to say, a step that will be optimal for the bilinear complexity of multiplication in a degree $n$ extension of $\mathbb{F}_{q}$, for any integer $n$.

Lemma 3.12. Let $n \geq \frac{1}{2}\left(q^{2}+1+\epsilon\left(q^{2}\right)\right)$ be an integer, with the same definition for $\epsilon$ as in Lemma 2.1. If $q=p^{r} \geq 4$, then there exists a step $F_{k, s} / \mathbb{F}_{q^{2}}$ of the tower $T_{2} / \mathbb{F}_{q^{2}}$ such that the following conditions are verified:

(1) there exists a place of $F_{k, s} / \mathbb{F}_{q^{2}}$ of degree $n$,

(2) $B_{1}\left(F_{k, s} / \mathbb{F}_{q^{2}}\right) \geq 2 n+g_{k, s}-1$.

Moreover, the first step for which both conditions (1) and (2) are verified is the first step for which (2) is verified.

Proof. Note that $n \geq 13$ since $q \geq 4$ and $n \geq \frac{1}{2}\left(q^{2}+1+2 q\right) \geq 12.5$. First, we prove that for $1 \leq k \leq n-2$ and $s \in\{0, \ldots, r\}$, there exists a place of $F_{k, s} / \mathbb{F}_{q^{2}}$ of degree $n$. Indeed, for such an integer $k$, one has $q^{n-k-1} \geq q>2 \times \frac{5}{3} \geq 2 \frac{q+1}{q-1}$, so $q^{n-k} p^{-s}>2 \frac{q+1}{q-1}$ since $1 \geq p^{-s} \geq q^{-1}$, which gives $2 q^{k-1}(q+1) p^{s}<q^{n-1}(q-1)$. Thus Lemma 3.4 iii) implies that $2 g_{k, s}+1 \leq q^{n-1}(q-1)$, which ensures that there exists a place of $F_{k, s} / \mathbb{F}_{q^{2}}$ of degree $n$. On the other hand, we prove that for $k \geq K(n)+1$, with $K(n):=\log _{q}\left(\frac{2 n}{(q+1)(q-2)}\right)$, condition (2) is satisfied. Indeed, for such integers $k$, one has $\frac{2 n}{(q+1)(q-2)} \leq q^{k-1}$, so $2 n-1 \leq q^{k-1}(q+1)(q-2) p^{s}$. Hence, one gets $2 n+q^{k-1}(q+1) p^{s}-1 \leq\left(q^{2}-1\right) q^{k-1} p^{s}$, which gives the result according to Lemma 3.4 iii) and Proposition 3.1 (3). To conclude, note that there exists at least one step $F_{k, s} / \mathbb{F}_{q^{2}}$ satisfying both conditions (1) and (2) since for $n \geq 13$ and $q \geq 4, n-K(n)-3 \geq 13-\left(\log _{4}(2 \cdot 13)\right)-3>1$. Moreover, we remark that condition (1) is satisfied from the step $F_{1,0} / \mathbb{F}_{q^{2}}$, so the first step for which both conditions (1) and (2) are verified is the first step for which (2) is verified.

This is a similar result for the tower $T_{3} / \mathbb{F}_{q}$ : 
Lemma 3.13. Let $n \geq \frac{1}{2}(q+1+\epsilon(q))$ be an integer, with the same definition for $\epsilon$ as in Lemma 2.1. If $q=p^{r}>5$, then there exists a step $G_{k, s} / \mathbb{F}_{q}$ of the tower $T_{3} / \mathbb{F}_{q}$ such that the following conditions are verified:

(1) there exists a place of $G_{k, s} / \mathbb{F}_{q}$ of degree $n$,

(2) $B_{1}\left(G_{k, s} / \mathbb{F}_{q}\right)+2 B_{2}\left(G_{k, s} / \mathbb{F}_{q}\right) \geq 2 n+g_{k, s}-1$.

Moreover, the first step for which both conditions (1) and (2) are verified is the first step for which (2) is verified.

Proof. Here we have $n \geq 7$ since $q \geq 7$ and $n \geq \frac{1}{2}(q+1+\epsilon(q)) \geq 6.5$. First, we prove that for $1 \leq k \leq \frac{n}{2}-2$ and $s \in\{0, \ldots, r\}$, there exists a place of $G_{k, s} / \mathbb{F}_{q}$ of degree $n$, by showing that $2 g_{k, s}+1 \leq q^{\frac{n-1}{2}}(\sqrt{q}-1)$. Indeed, the function

$$
q \mapsto \frac{\sqrt{q}-1}{q+1} \cdot q^{\frac{n-1}{2}-k}
$$

is increasing, so one has $\frac{\sqrt{q}-1}{q+1} \cdot q^{\frac{n-1}{2}-k} \geq \frac{\sqrt{7}-1}{8} \cdot 7^{\frac{n-1}{2}-k}$ since $q \geq 7$. Thus for any $k \leq \frac{n}{2}-2$, we get $\frac{\sqrt{q}-1}{q+1} \cdot q^{\frac{n-1}{2}-k} \geq \frac{7^{\frac{3}{2}}(\sqrt{7}-1)}{8}>2$. It follows that $2 q^{k}(q+1)<$ $q^{\frac{n-1}{2}}(\sqrt{q}-1)$, so $2 q^{k-1}(q+1) p^{s}<q^{\frac{n-1}{2}}(\sqrt{q}-1)$ since $p^{s} \leq q$, and we get $2 q^{k-1}(q+$ 1) $p^{s}+1 \leq q^{\frac{n-1}{2}}(\sqrt{q}-1)$ which ensures that there exists a place of $F_{k, s} / \mathbb{F}_{q^{2}}$ of degree $n$, according to Lemma 3.4 iii). On the other hand, we can proceed as the preceding proof to prove that for $k \geq K(n)+1$, with $K(n):=\log _{q}\left(\frac{2 n}{(q+1)(q-2)}\right)$, condition (2) is satisfied. To conclude, note that there exists at least one step $G_{k, s} / \mathbb{F}_{q}$ satisfying both conditions (1) and (2) since for $n \geq 7$ and $q \geq 7, \frac{n}{2}-$ $K(n)-3 \geq \frac{7}{2}-\log _{7}\left(\frac{2 \times 7}{8 \times 5}\right)-3>1$. Moreover, we remark that condition (1) is satisfied from the step $G_{1,0} / \mathbb{F}_{q}$, so the first step for which both conditions (1) and (2) are verified is the first step for which (2) is verified.

In the special case where $q=4$, condition (2) needs to be slightly stronger:

Lemma 3.14. Let $n \geq 10$ be an integer. If $q=p^{2}=4$, then there exists a step $G_{k, s} / \mathbb{F}_{4}$ of the tower $T_{3} / \mathbb{F}_{4}$ such that the following conditions are verified:

(1) there exists a place of $G_{k, s} / \mathbb{F}_{4}$ of degree $n$,

(2) $B_{1}\left(G_{k, s} / \mathbb{F}_{4}\right)+2 B_{2}\left(G_{k, s} / \mathbb{F}_{4}\right) \geq 2 n+g_{k, s}+2$.

Moreover, the first step for which both conditions (1) and (2) are verified is the first step for which (2) is verified.

Proof. We can proceed as in the previous proof with minor changes. Indeed, we first have that $2 g_{k, s}+1 \leq q^{\frac{n-1}{2}}(\sqrt{q}-1)$ for $1 \leq k \leq \frac{n-9 / 2}{2}$ and $s \in\{0,1\}$, since in this case $\frac{\sqrt{q}-1}{q+1} \cdot q^{\frac{n-1}{2}-k}=\frac{1}{5} 2^{n-1-2 k} \geq \frac{2^{7 / 2}}{5}>2$, which proves that condition (1) is verified according to Lemma 3.4 iii). Moreover, condition (2) is satisfied for $k \geq K(n)+1$ with $K(n):=\log _{4}\left(\frac{2 n+2}{(q+1)(q-2)}\right)$, and one can check that $\frac{n}{2}-K(n)-$ $\frac{9}{4}-1 \geq \frac{10}{2}-\frac{9}{4}-\log _{4}\left(\frac{20}{10}\right)>1$.

This is a similar result for the tower $T_{4} / \mathbb{F}_{2}$ :

Lemma 3.15. For any integer $n \geq 12$ there exists a step $H_{k, s} / \mathbb{F}_{2}$ of the tower $T_{4} / \mathbb{F}_{2}$, with genus $g_{k, s} \geq 2$, such that both of the following conditions are verified:

(1) there exists a place of degree $n$ in $H_{k, s} / \mathbb{F}_{2}$, 
(2) $B_{1}\left(H_{k, s} / \mathbb{F}_{2}\right)+2 B_{2}\left(H_{k, s} / \mathbb{F}_{2}\right)+4 B_{4}\left(H_{k, s} / \mathbb{F}_{2}\right) \geq 2 n+g_{k, s}+5$.

Moreover, the first step for which both conditions (1) and (2) are verified is the first step for which (2) is verified.

Proof. According to [8, Lemma 2.6], if $n \geq 12$, then there exists a step $H_{k, s} / \mathbb{F}_{2}$ of the tower $T_{4} / \mathbb{F}_{2}$, with $k \geq 2$ (so, in particular, $g_{k, s} \geq g_{2}=6$ ) such that there exists a place of $H_{k, s} / \mathbb{F}_{2}$ of degree $n$ and

$$
B_{1}\left(H_{k, s} / \mathbb{F}_{2}\right)+2 B_{2}\left(H_{k, s} / \mathbb{F}_{2}\right)+4 B_{4}\left(H_{k, s} / \mathbb{F}_{2}\right) \geq 2 n+2 g_{k, s}+7 .
$$

Thus we get the result since $2 n+2 g_{k, s}+7 \geq 2 n+g_{k, s}+5$.

This is a similar result for the tower $T / \mathbb{F}_{p^{2}}$ :

Lemma 3.16. Let $p \geq 3$ and $n \geq \frac{1}{2}\left(p^{2}+1+\epsilon\left(p^{2}\right)\right)$, with the same definition for $\epsilon$ as in Lemma 2.1. There exists a step $L_{k} / \mathbb{F}_{p^{2}}$ of the tower $T / \mathbb{F}_{p^{2}}$, with genus $g_{k} \geq 2$, such that the following conditions are verified:

(1) there exists a place of $L_{k} / \mathbb{F}_{p^{2}}$ of degree $n$,

(2) $B_{1}\left(L_{k} / \mathbb{F}_{p^{2}}\right) \geq 2 n+g_{k}-1$.

Moreover, the first step for which both conditions (1) and (2) are verified is the first step for which (2) is verified.

Proof. Note that $n \geq \frac{1}{2}\left(3^{2}+1+2 \cdot 3\right)=8$. We first prove that for all integers $k$ such that $2 \leq k \leq n-2$, we have $2 g_{k}+1 \leq p^{n-1}(p-1)$, so condition (2) is satisfied. Indeed, for such an integer $k$, one has $2^{k+1} \leq 2^{n-1}<p^{n-1}$, since $p>2$. Thus $2 \cdot 2^{k+1}<p^{n-1}(p-1)$ since $2 \leq p-1$ and we get the result from Lemma 3.9 ii).

We prove now that for $k \geq \log _{2}\left(\frac{n}{2}\right)$, condition (2) is verified. Indeed, for such an integer $k$, we have $2^{k+2} \geq 2 n$, so $2^{k+2} \geq 2 n-2 \cdot 2^{\frac{k+1}{2}}$. Hence we get $2^{k+1}(p-2) \geq$ $2 n-2 \cdot 2^{\frac{k+1}{2}}$ since $p \geq 3$ and then we obtain $2^{k+1}(p-1) \geq 2 n+2^{k+1}-2 \cdot 2^{\frac{k+1}{2}}$. Thus we have $B_{1}\left(L_{k} / \mathbb{F}_{p^{2}}\right) \geq 2 n+g_{k}-1$ according to bound (9) and Lemma $\left.3.9 \mathrm{i}\right)$.

Hence, we have proved that for any integers $n \geq 8$ and $k \geq 2$ such that $\log _{2}\left(\frac{n}{2}\right) \leq$ $k \leq n-2$, both conditions (1) and (2) are verified. Moreover, note that for any $n \geq 8$, there exists an integer $k \geq 2$ in the interval $\left[\log _{2}\left(\frac{n}{2}\right) ; n-2\right]$ since $n-2-$ $\log _{2}\left(\frac{n}{2}\right) \geq 6-\log _{2}(4)>1$. To conclude, we remark that condition (1) is satisfied from the step $L_{0} / \mathbb{F}_{p^{2}}$, so the first step for which both conditions (1) and (2) are verified is the first step for which (2) is verified; moreover, for $k \geq 2, g_{k} \geq g_{2}=3$.

This is a similar result for the tower $T / \mathbb{F}_{p}$ :

Lemma 3.17. Let $p>5$ and $n \geq \frac{1}{2}(p+1+\epsilon(p))$, with the same definition for $\epsilon$ as in Lemma 2.1. There exists a step $L_{k} / \mathbb{F}_{p}$ of the tower $T / \mathbb{F}_{p}$, with genus $g_{k} \geq 2$, such that the following conditions are verified:

(1) there exists a place of $L_{k} / \mathbb{F}_{p}$ of degree $n$,

(2) $B_{1}\left(L_{k} / \mathbb{F}_{p}\right)+2 B_{2}\left(L_{k} / \mathbb{F}_{p}\right) \geq 2 n+g_{k}-1$.

Moreover, the first step for which both conditions (1) and (2) are verified is the first step for which (2) is verified.

Proof. Note that $n \geq \frac{1}{2}(7+1+\epsilon(7))=7$. We first prove that for all integers $k$ such that $2 \leq k \leq n-3$, we have $2 g_{k}+1 \leq p^{\frac{n-1}{2}}(\sqrt{p}-1)$, so condition (1) is satisfied. Indeed, for such an integer $k$, one has $2^{k+2} \leq 2^{n-1}=4^{\frac{n-1}{2}}$, so $2 \cdot 2^{k+1}<p^{\frac{n-1}{2}}$ since 
$p>4$. Hence we get $2 \cdot 2^{k+1}<p^{\frac{n-1}{2}}(\sqrt{p}-1)$, which gives the result from Lemma 3.9 ii).

On the other hand, we proceed as the preceding proof to prove that for $k \geq$ $\log _{2}\left(\frac{n}{2}\right)$, condition (2) is verified. Moreover, note that for any $n \geq 7$, there exists an integer $k \geq 2$ in the interval $\left[\log _{2}\left(\frac{n}{2}\right) ; n-3\right]$ since $n-3-\log _{2}\left(\frac{n}{2}\right) \geq 4-\log _{2}(3.5)>$ 1. To conclude, we remark that condition (1) is satisfied from the step $L_{0} / \mathbb{F}_{p}$, so the first step for which both conditions (1) and (2) are verified is the first step for which (2) is verified; moreover, for $k \geq 2, g_{k} \geq g_{2}=3$.

This is a similar result for the tower $T / \mathbb{F}_{p}$ for $p=3$ or 5 :

Lemma 3.18. If $p=5$ and $n \geq \frac{1}{2}(5+1+\epsilon(5))=5$ (according to the definition of $\epsilon$ in Lemma 2.1) or $p=3$ and $n \geq 11$, then there exists a step $L_{k} / \mathbb{F}_{p}$ of the tower $T / \mathbb{F}_{p}$, with genus $g_{k} \geq 2$, such that the following conditions are verified:

(1) there exists a place of $L_{k} / \mathbb{F}_{p}$ of degree $n$,

(2) $B_{1}\left(L_{k} / \mathbb{F}_{p}\right)+2 B_{2}\left(L_{k} / \mathbb{F}_{p}\right) \geq 2 n+g_{k, s}+2$.

Moreover, the first step for which both conditions (1) and (2) are verified is the first step for which (2) is verified.

Proof. We first consider the case $p=5$ and $n \geq 5$. Since $p>4$, the first part of the preceding proof shows that for all integers $k$ such that $2 \leq k \leq n-3$, we have $2 g_{k}+1 \leq p^{\frac{n-1}{2}}(\sqrt{p}-1)$, so condition (1) is satisfied. Now, we prove that for $k \geq \log _{2}\left(\frac{n}{3}\right)$, condition (2) is satisfied. Indeed, for such an integer $k$, one has

$$
2^{k+1}(p-2)+2^{\frac{k+3}{2}} \geq 2 n+2 \sqrt{\frac{2 n}{3}}>2 n+3
$$

since $n \geq 5$. Thus we get $2^{k+1}(p-1)>2 n+\left(2^{k+1}-2^{\frac{k+3}{2}}+1\right)+2$, which gives the result according to bound (9) and Lemma 3.9 i). Hence, we have proved that for any integers $n \geq 5$ and $k \geq 2$ such that $\log _{2}\left(\frac{n}{3}\right) \leq k \leq n-3$, both conditions (1) and (2) are verified. Moreover, note that for any $n \geq 5$, there exists an integer $k \geq 2$ in the interval $\left[\log _{2}\left(\frac{n}{3}\right) ; n-3\right]$ since $n-3-\log _{2}\left(\frac{n}{3}\right) \geq 2-\log _{2}\left(\frac{n}{3}\right)>1$. To conclude, we remark that condition (1) is satisfied from the step $L_{0} / \mathbb{F}_{p^{2}}$, so the first step for which both conditions (1) and (2) are verified is the first step for which (2) is verified; moreover, for $k \geq 2, g_{k} \geq g_{2}=3$.

Now we consider the case $p=3$ and $n \geq 11$. We first prove that for all integers $k$ such that $2 \leq k \leq \log _{2}\left(3^{\frac{n-1}{2}}\right)-3$, we have $2 g_{k}+1 \leq 3^{\frac{n-1}{2}}(\sqrt{3}-1)$, so condition (1) is satisfied. Indeed, for such an integer $k$, one has $2^{k+3} \leq 3^{\frac{n-1}{2}}$, so $2 \cdot 2^{k+1} \leq \frac{1}{2} \cdot 3^{\frac{n-1}{2}}<3^{\frac{n-1}{2}}(\sqrt{3}-1)$ which gives the result from Lemma 3.9 ii). On the other hand, we prove that for $k \geq \log _{2}(n)$, condition (2) is satisfied. Indeed for such an integer $k$, one has $2^{k+1}(p-2)+2^{\frac{k+3}{2}}=2^{k+1}+2^{\frac{k+3}{2}} \geq 2 n+2 \sqrt{2 n}>2 n+3$ since $n \geq 11$. Thus we get $2^{k+1}(p-1)>2 n+\left(2^{k+1}-2^{\frac{k+3}{2}}+1\right)+2$, which gives the result according to bound (9) and Lemma 3.9]i). Hence, we have proved that for any integers $n \geq 11$ and $k \geq 2$ such that $\log _{2}(n) \leq k \leq \log _{2}\left(3^{\frac{n-1}{2}}\right)-3$, both conditions (1) and (2) are verified. Moreover, note that for any $n \geq 11$, there exists an integer $k \geq 2$ in the interval $\left[\log _{2}(n) ; \log _{2}\left(3^{\frac{n-1}{2}}\right)-3\right]$ since $\log _{2}\left(3^{\frac{n-1}{2}}\right)-3-\log _{2}(n) \geq$ $\log _{2}\left(3^{5}\right)-3-\log _{2}(11)>1$. To conclude, we remark that condition (1) is satisfied from the step $L_{0} / \mathbb{F}_{p^{2}}$, so the first step for which both conditions (1) and (2) are verified is the first step for which (2) is verified; moreover, for $k \geq 2, g_{k} \geq g_{2}=3$. 


\section{NEW UNIFORM BOUNDS FOR THE TENSOR RANK}

Theorem 4.1. For any integer $n \geq 2$, we have

$$
\mu_{2}(n) \leq \frac{189}{22} n+18
$$

In particular, it follows that $\mu_{2}(n) \leq C_{2} n$ with

$$
C_{2}=9.54 \text {. }
$$

Proof. Let $q:=p^{2}=4$ and $n \geq 2$. We apply the general method described in Section 2.1 on the tower $T_{4} / \mathbb{F}_{q}$ with $d=4, \gamma_{2,4} \leq \frac{3}{2}$ (see Proof of Corollary 2.4) and $\lambda:=\frac{4 \gamma_{2,4}}{\mu_{2}^{\text {slm }}(4)} \leq \frac{2}{3}$, since $\mu_{2}^{\text {sym }}(4)=9$.

We set $X=n_{0}^{k, s}+\frac{1}{2}\left(D_{k, s}-3\right)$ where $D_{k, s}=\frac{3}{2} p^{s+1} q^{k-1}$. Lemmas 3.7 and 3.15 ensure that hypotheses (A) to (E) are satisfied, so we have:

$$
\begin{aligned}
\Phi(X) & =\frac{2 \mu_{q}^{\mathrm{sym}}(d)}{d}\left(1+\frac{g\left(H_{k, s+1}\right)}{2 X}\right) X+\frac{\mu_{q}^{\mathrm{sym}}(d)}{d}\left(\alpha_{q}+d-1\right) \\
& =\frac{9}{2}\left(1+\frac{g\left(H_{k, s+1}\right)}{2 X}\right) X+18 .
\end{aligned}
$$

From Lemmas 3.4 iii) and 3.8 it follows that

$$
\begin{aligned}
\frac{g\left(H_{k, s+1}\right)}{2 X} & \leq \frac{q^{k-1}(q+1) p^{s+1}}{2 n_{0}^{k, s}+D_{k, s}-3} \\
& \leq \frac{q^{k-1}(q+1) p^{s+1}}{5 p^{s+1} q^{k-1}-5+\frac{3}{2} p^{s+1} q^{k-1}-3} \\
& =\frac{q+1}{\frac{13}{2}-\frac{8}{q^{k-1} p^{s+1}}} .
\end{aligned}
$$

Since $k \geq 2$, one has $\frac{g\left(H_{k, s+1}\right)}{2 X} \leq \frac{10}{11}$ which leads to $\mu_{q}(n) \leq \frac{9}{2}\left(1+\frac{10}{11}\right) n+18$ and gives the result.

The new constant $C_{2}$ follows on one hand from the values in [13, Table 1], which gives that for $n \leq 18, \mu_{2}^{\text {sym }}(n) \leq 4 n$. On the other hand, applying our new bound for $\mu_{2}(n)$ for $n \geq 19$ we get

$$
\frac{\mu_{2}(n)}{n} \leq \frac{189}{22}+\frac{18}{n} \leq \frac{189}{22}+\frac{18}{19} \leq 9.54
$$

Theorem 4.2. Let $p$ be a prime and $q:=p^{r}$. For any $n \geq 2$, we have:

(a) if $q \geq 4$, then

$$
\mu_{q^{2}}(n) \leq 2\left(1+\frac{p}{q-2+(p-1) \frac{q}{q+1}}\right) n-1,
$$

(b) if $p \geq 3$, then

$$
\mu_{p^{2}}(n) \leq 2\left(1+\frac{2}{p-1}\right) n-1
$$

(c) if $q>5$, then

$$
\mu_{q}(n) \leq 3\left(1+\frac{p}{q-2+(p-1) \frac{q}{q+1}}\right) n
$$


(d) if $p>5$, then

$$
\mu_{p}(n) \leq 3\left(1+\frac{2}{p-1}\right) n
$$

Proof. (a) Let $n \geq \frac{1}{2}\left(q^{2}+1+\epsilon\left(q^{2}\right)\right)$. We apply the general method described in Section 2.1 on the tower $T_{2} / \mathbb{F}_{q^{2}}$ with $d=1, \gamma_{q^{2}, 1} \leq 1$ (see the proof of Corollary 2.3) and $\lambda:=\frac{\gamma_{q^{2}, 1}}{\mu_{q^{2}}^{\text {sym }}(1)} \leq 1$.

We set $X=n_{0}^{k, s}+\frac{1}{2} D_{k, s}$ where $D_{k, s}=(p-1) p^{s} q^{k}$. Lemmas 3.5 and 3.12 ensure that hypotheses (A) to (E) are satisfied. Note that we can always choose a step $F_{k, s+1}$ with $k \geq 4$ (so in particular $g_{k, s+1} \geq 2$ ), even if doing so we may have a non-optimal bound for some small $n$.

Thus we have

$$
\Phi(X)=2\left(1+\frac{g\left(F_{k, s+1}\right)}{2 X}\right) X-1 .
$$

From Lemmas 3.4 iii) and 3.6 it follows that

$$
\begin{aligned}
\frac{g\left(F_{k, s+1}\right)}{2 X} & \leq \frac{q^{k-1}(q+1) p^{s+1}}{2 n_{0}^{k, s}+D_{k, s}} \\
& \leq \frac{q^{k-1}(q+1) p^{s+1}}{(q+1) q^{k-1} p^{s}(q-2)+(p-1) p^{s} q^{k}} \\
& =\frac{p}{q-2+(p-1) \frac{q}{q+1}},
\end{aligned}
$$

which gives the result.

(b) Let $n \geq \frac{1}{2}\left(p^{2}+1+\epsilon\left(p^{2}\right)\right)$. We apply the general method described in Section 2.1 on the tower $T / \mathbb{F}_{p^{2}}$ with $d=1, \gamma_{p^{2}, 1} \leq 1$ and $\lambda:=\frac{\gamma_{p^{2}, 1}}{\mu_{p^{2}}(1)} \leq 1$.

We set $X=n_{0}^{k}+\frac{1}{2} D_{k}$ where $D_{k}=2^{k+1}-2^{\frac{k+1}{2}}$. Lemmas 3.10 and 3.16 ensure that hypotheses (A) to (E) are satisfied.

Thus we have:

$$
\Phi(X)=2\left(1+\frac{g\left(L_{k+1}\right)}{2 X}\right) X-1 .
$$

From Lemmas 3.9 ii) and 3.11 it follows that

$$
\begin{aligned}
\frac{g\left(L_{k+1}\right)}{2 X} & \leq \frac{2^{k+2}}{2 n_{0}^{k, s}+D_{k, s}} \\
& \leq \frac{2^{k+2}}{2^{k+1}(p-2)+2^{\frac{k+3}{2}}+2^{k+1}-2^{\frac{k+1}{2}}} \\
& =\frac{2}{p-1+2^{-\frac{k-1}{2}}-2^{-\frac{k+1}{2}}}
\end{aligned}
$$

which gives the result, since $2^{-\frac{k-1}{2}}-2^{-\frac{k+1}{2}} \geq 0$.

(c) Let $n \geq \frac{1}{2}(q+1+\epsilon(q))$. We apply the general method described in Section 2.1 on the tower $T_{3} / \mathbb{F}_{q}$ with $d=2, \gamma_{q, 2} \leq \frac{1}{2}$ (see the proof of Corollary 2.3) and $\lambda:=\frac{2 \gamma_{q, 2}}{\mu_{q}^{\text {sym }}(2)} \leq \frac{1}{3}$ since $\mu_{q}^{\text {sym }}(2) \geq 3$.

We set $X=n_{0}^{k, s}+\frac{1}{2}\left(D_{k, s}-1\right)$ where $D_{k, s}=(p-1) p^{s} q^{k}$. Lemmas 3.5 and 3.13 ensure that hypotheses (A) to (E) are satisfied. Note that we can always 
choose a step $F_{k, s+1}$ with $k \geq 4$ (so in particular $g_{k, s+1} \geq 2$ ), even if doing so we may have a non-optimal bound for some small $n$.

Thus we have:

$$
\Phi(X)=3\left(1+\frac{g\left(G_{k, s+1}\right)}{2 X}\right) X .
$$

We proceed as in (a) to get $\frac{g\left(G_{k, s+1}\right)}{2 X} \leq \frac{p}{q-2+(p-1) \frac{q}{q+1}}$ which gives the result. (Note that $\lambda \leq 1$ so Lemma 3.5 implies that hypothesis (D) of Section 2.1 is satisfied.)

(d) Let $n \geq \frac{1}{2}(p+1+\epsilon(p))$. We apply the general method described in Section 2.1 on the tower $T / \mathbb{F}_{p}$ with $d=2, \gamma_{p, 2} \leq \frac{1}{2}$ (see the proof of Corollary 2.3) and $\lambda:=\frac{2 \gamma_{p, 2}}{3} \leq \frac{1}{3}$.

We set $X=n_{0}^{k}+\frac{1}{2}\left(D_{k}-1\right)$ where $D_{k}=2^{k+1}-2^{\frac{k+1}{2}}$. Lemmas 3.10 and 3.17 ensure that hypotheses (A) to (E) are satisfied.

Thus we have

$$
\Phi(X)=3\left(1+\frac{g\left(L_{k+1}\right)}{2 X}\right) X .
$$

We proceed as in (b) to get $\frac{g\left(L_{k+1}\right)}{2 X} \leq \frac{2}{p-1}$ which gives the result. (Note that $\lambda \leq 1$, so Lemma 3.10 implies that hypothesis (D) of Section 2.1] is satisfied.)

Theorem 4.3. For any $n \geq 2$, we have

$$
\mu_{3}(n) \leq 6 n, \quad \mu_{4}(n) \leq \frac{87}{19} n, \quad \text { and } \quad \mu_{5}(n) \leq \frac{9}{2} n .
$$

Proof. For the bounds over $\mathbb{F}_{3}$ and $\mathbb{F}_{5}$, we proceed as in the proof of Theorem 4.2 (d), since Lemma 3.18 ensures that the method is still valid in this cases. Thus we get

$$
\mu_{p}(n) \leq 3\left(1+\frac{2}{p-1}\right) n
$$

Note that with our method, we prove the bound for $\mu_{3}(n)$ for $n \geq 11$ according to Lemma 3.18, but that this bound holds also for $n \leq 10$, according to Table 1 in $[13$.

The bound over $\mathbb{F}_{4}$ is obtained for $n \geq 10$ with the same reasoning as in the proof of Theorem 4.2 (ㄷ) : let $q:=4$ and $n \geq 10>\frac{1}{2}(q+1+\epsilon(q))$, we apply the general method described in Section 2.1 on the tower $T_{3} / \mathbb{F}_{4}$ with $d=2, \gamma_{4,2} \leq 1$ (see the proof of Corollary 2.3) and $\lambda:=\frac{2 \gamma_{4,2}}{\mu_{4}^{\text {sym }}(2)} \leq \frac{2}{3}$ since $\mu_{4}^{\text {sym }}(2) \geq 3$. We set $X=n_{0}^{k, s}+\frac{1}{2}\left(D_{k, s}-1\right)$ where $D_{k, s}=(p-1) p^{s} q^{k-1}$. Lemmas 3.5 and 3.14 ensure that hypotheses (A) to (E) are satisfied. Note that we can always choose a step $F_{k, s+1}$ with $k \geq 4$ (so in particular $g_{k, s+1} \geq 2$ ), even if doing so we may have a non-optimal bound for some small $n$. Thus we have

$$
\Phi(X)=3\left(1+\frac{g\left(G_{k, s+1}\right)}{2 X}\right) X
$$

which gives $\frac{g\left(G_{k, s+1}\right)}{2 X} \leq \frac{p}{q-2+(p-1) \frac{q}{q+1}}$. (Note that $\lambda \leq 1$ so Lemma 3.5 implies that hypothesis (D) of Section 2.1 is satisfied.) To conclude, we remark that our bound 
is still valid for $\mu_{4}(n)$ when $4.5=\frac{1}{2}(q+1+\epsilon(q)) \leq n<10$ according to the known estimates for $\mu_{4}^{\mathrm{sym}}(n)$ (recalled in [13, Table 1]).

\section{Asymptotic Bounds}

So far we gave upper bounds for the tensor rank of multiplication that hold uniformly for any extension of finite fields. Now, introducing the quantity

$$
M_{q}=\limsup _{n \rightarrow \infty} \frac{\mu_{q}(n)}{n}
$$

and letting the degree of the extension go to infinity, these bounds then turn into the following asymptotic estimates:

Proposition 5.1. We have

$$
M_{2} \leq \frac{189}{22} \approx 8.591, \quad M_{3} \leq 6, \quad M_{4} \leq \frac{87}{19} \approx 4.579, \quad M_{5} \leq 4.5,
$$

and for $p$ a prime and $q=p^{r}$,

(a) if $q \geq 4$, then $M_{q^{2}} \leq 2\left(1+\frac{p}{q-2+(p-1) \frac{q}{q+1}}\right)$,

(b) if $p \geq 3$, then $M_{p^{2}} \leq 2\left(1+\frac{2}{p-1}\right)$,

(c) if $q>5$, then $M_{q} \leq 3\left(1+\frac{p}{q-2+(p-1) \frac{q}{q+1}}\right)$,

(d) if $p>5$, then $M_{p} \leq 3\left(1+\frac{2}{p-1}\right)$.

Proof. Let $n \rightarrow \infty$ in Theorems 4.14 .2 , and 4.3 .

It is interesting to compare these asymptotic bounds with other known similar results, such as the ones in [12] and [5]. We see the bound on $M_{2}$ in Proposition 5.1 is less sharp than the one in [12, while the bounds on $M_{3}, M_{4}$, and $M_{5}$ are better. Moreover, one can check that the general bounds on $M_{q^{2}}$ and $M_{q}$ established in [5] are better than those given by (a) and (c).

However, in such a comparison, one should keep in mind other features of these various bounds. On one hand, the bounds in [12] and [5] hold not only for the general bilinear complexity, but also for the symmetric bilinear complexity. On the other hand, the constructions leading to Proposition 5.1 were not aimed solely at maximizing asymptotics:

- they give uniform bounds, that hold for any given extension of finite fields (so, not only asymptotically),

- they come from towers of curves given by explicit equations, so at least in principle, it should be possible to write explicitly the multiplication algorithms reaching these bounds.

Now, if one relaxes these last two conditions, it is possible to give substantially better asymptotic bounds, especially for $q$ small. For this we will borrow the following lemma from [12] (with a very slight modification):

Lemma 5.2 (compare 12, Lemma IV.4). Let $q$ be a prime power and $t \geq 1$ an integer such that $q^{t}$ is a square (so $q$ itself is a square, or $t$ is even). Then there exists a family $\left(F_{s} / \mathbb{F}_{q}\right)_{s \geq 1}$ of function fields such that, as $s$ goes to infinity, we have:

(i) $g_{s} \rightarrow \infty$,

(ii) $g_{s+1} / g_{s} \rightarrow 1$, 
(iii) $B_{t}\left(F_{s}\right) / g_{s} \rightarrow\left(q^{t / 2}-1\right) / t$,

where $g_{s}$ is the genus of $F_{s} / \mathbb{F}_{q}$.

For the details of the proof we refer to 12 , where it is in fact credited to Elkies, who proceeded by modifying the construction of Shimura curves previously introduced in 20.

As a matter of fact, the version of the lemma originally stated in [12] requires $t$ even, while we allow $t$ odd provided $q$ is a square. However, our increased generality is only apparent, because it is readily seen that the aforementioned proof of Elkies also gives the version we stated. Alternatively, when $q$ is a square, we can replace $q$ and $t$ with $q^{1 / 2}$ and $2 t$ to reduce to the case $t$ even, and conclude with a base field extension argument.

Theorem 5.3. Let $q$ be a prime power and $t \geq 1$ an integer such that $q^{t} \geq 9$ is a square. Then

$$
M_{q} \leq \frac{2 \mu_{q}(t)}{t}\left(1+\frac{1}{q^{t / 2}-2}\right) .
$$

Proof. Let $\left(F_{s} / \mathbb{F}_{q}\right)_{s \geq 1}$ be the family of function fields given by Lemma 5.2 for $q$ and $t$. Given an integer $n$, let $s(n)$ be the smallest integer such that

$$
t B_{t}\left(F_{s(n)} / \mathbb{F}_{q}\right)-g_{s(n)} \geq 2 n+8 .
$$

Such an integer exists because of conditions (i) and (iii) in Lemma 5.2 and our hypothesis $q^{t} \geq 9$, and it goes to infinity with $n$. More precisely, minimality of $s(n)$ and conditions (iii) and (ii) give, respectively:

- $t B_{t}\left(F_{s(n)} / \mathbb{F}_{q}\right)-g_{s(n)} \geq 2 n+8>t B_{t}\left(F_{s(n)-1} / \mathbb{F}_{q}\right)-g_{s(n)-1}$,

- $t B_{t}\left(F_{s(n)} / \mathbb{F}_{q}\right)=\left(q^{t / 2}-1\right) g_{s(n)}+o\left(g_{s(n)}\right)$,

- $g_{s(n)-1}=g_{s(n)}+o\left(g_{s(n)}\right)$,

hence the estimate

$$
\left(q^{t / 2}-2\right) g_{s(n)}+o\left(g_{s(n)}\right)=2 n+o(n)
$$

which can be restated finally as

$$
g_{s(n)}=\frac{2 n}{q^{t / 2}-2}+o(n)
$$

and

$$
B_{t}\left(F_{s(n)} / \mathbb{F}_{q}\right)=\frac{2 n}{t}\left(1+\frac{1}{q^{t / 2}-2}\right)+o(n) .
$$

The estimate on $g_{s(n)}$ implies $2 g_{s(n)}+1 \leq q^{(n-1) / 2}\left(q^{1 / 2}-1\right)$ as soon as $n$ is big enough. We can then use Theorem 1.3 with $F_{s(n)} / \mathbb{F}_{q}$, setting $m=n, l=1$, $N_{t}=n_{t, 1}=B_{t}\left(F_{s(n)} / \mathbb{F}_{q}\right)$, and $n_{d, u}=0$ for all other values of $d$ and $u$. This gives

$$
\mu_{q}(n) \leq \mu_{q}(t) B_{t}\left(F_{s(n)} / \mathbb{F}_{q}\right)
$$

and the conclusion follows.

Corollary 5.4. We have:

$$
\begin{aligned}
& M_{2} \leq 35 / 6 \approx 5.833 \\
& M_{3} \leq 36 / 7 \approx 5.143, \\
& M_{4} \leq 30 / 7 \approx 4.286 .
\end{aligned}
$$


Proof. Apply Theorem 5.3 with $q=2, t=6, \mu_{2}(6) \leq 15$; with $q=3, t=4$, $\mu_{3}(4) \leq 9$; and with $q=4, t=4, \mu_{4}(4) \leq 8$.

Corollary 5.5. For any $q \geq 3$ we have $M_{q} \leq 3\left(1+\frac{1}{q-2}\right)$. In particular,

$$
\begin{gathered}
M_{5} \leq 4, \\
M_{7} \leq 3.6, \\
M_{8} \leq 3.5 .
\end{gathered}
$$

Proof. Apply Theorem 5.3 with $t=2, \mu_{q}(2)=3$.

\section{REFERENCES}

[1] Stéphane Ballet, Curves with many points and multiplication complexity in any extension of $\mathbf{F}_{q}$, Finite Fields Appl. 5 (1999), no. 4, 364-377, DOI 10.1006/ffta.1999.0255. MR.1711833 (2001d:11065)

[2] Stéphane Ballet, Low increasing tower of algebraic function fields and bilinear complexity of multiplication in any extension of $\mathbb{F}_{q}$, Finite Fields Appl. 9 (2003), no. 4, 472-478, DOI 10.1016/S1071-5797(03)00026-1. MR2007465 (2004m:11191)

[3] Stéphane Ballet, On the tensor rank of the multiplication in the finite fields, J. Number Theory 128 (2008), no. 6, 1795-1806, DOI 10.1016/j.jnt.2007.06.010. MR2419195(2009b:11103)

[4] Stéphane Ballet and Jean Chaumine, On the bounds of the bilinear complexity of multiplication in some finite fields, Appl. Algebra Engrg. Comm. Comput. 15 (2004), no. 3-4, 205-211, DOI 10.1007/s00200-004-0155-7. MR2104295 (2005g:11253)

[5] Stéphane Ballet, Jean Chaumine, and Julia Pieltant, Shimura modular curves and asymptotic symmetric tensor rank of multiplication in any finite field, In Traian Muntean, Dimitrios Poulakis, and Robert Rolland, editors, Algebraic Informatics - 5th International Conference, CAI 2013, Porquerolles, France, September 3-6, 2013, Proceedings, volume 8080 of Lecture Notes in Computer Science, pp. 160-172. Springer, 2013.

[6] Stéphane Ballet and Dominique Le Brigand, On the existence of non-special divisors of degree $g$ and $g-1$ in algebraic function fields over $\mathbb{F}_{q}$, J. Number Theory 116 (2006), no. 2, 293-310, DOI 10.1016/j.jnt.2005.04.009. MR2195927 (2006i:11136)

[7] Stéphane Ballet, Dominique Le Brigand, and Robert Rolland, On an application of the definition field descent of a tower of function fields (English, with English and French summaries), Arithmetics, geometry, and coding theory (AGCT 2005), Sémin. Congr., vol. 21, Soc. Math. France, Paris, 2010, pp. 187-203. MR2856567(2012j:11220)

[8] Stéphane Ballet and Julia Pieltant, On the tensor rank of multiplication in any extension of $\mathbb{F}_{2}$, J. Complexity 27 (2011), no. 2, 230-245, DOI 10.1016/j.jco.2011.01.008. MR2776494 (2012c:11266)

[9] Stéphane Ballet and Robert Rolland, Multiplication algorithm in a finite field and tensor rank of the multiplication, J. Algebra 272 (2004), no. 1, 173-185, DOI 10.1016/j.jalgebra.2003.09.031. MR2029030 (2004i:11148)

[10] Stéphane Ballet and Robert Rolland, Families of curves over any finite field attaining the generalized Drinfeld-Vladut bound (English, with English and French summaries), Actes de la Conférence "Théorie des Nombres et Applications", Publ. Math. Besançon Algèbre Théorie Nr., Presses Univ. Franche-Comté, Besançon, 2011, pp. 5-18. MR2894265

[11] Ulrich Baum and Mohammad Amin Shokrollahi, An optimal algorithm for multiplication in $\mathbf{F}_{256} / \mathbf{F}_{4}$, Appl. Algebra Engrg. Comm. Comput. 2 (1991), no. 1, 15-20, DOI 10.1007/BF01810851. MR1209240 (94a:11191)

[12] Ignacio Cascudo, Ronald Cramer, Chaoping Xing, and An Yang, Asymptotic bound for multiplication complexity in the extensions of small finite fields, IEEE Trans. Inform. Theory $\mathbf{5 8}$ (2012), no. 7, 4930-4935, DOI 10.1109/TIT.2011.2180696. MR2949863

[13] Murat Cenk and Ferruh Özbudak, On multiplication in finite fields, J. Complexity 26 (2010), no. 2, 172-186, DOI 10.1016/j.jco.2009.11.002. MR2607731(2011b:68079)

[14] David Chudnovsky and Gregory Chudnovsky, Algebraic complexities and algebraic curves over finite fields, Journal of Complexity, 4:285-316, 1988. 
[15] Hans F. de Groote, Characterization of division algebras of minimal rank and the structure of their algorithm varieties, SIAM J. Comput. 12 (1983), no. 1, 101-117, DOI 10.1137/0212007. MR687805 (84e:68036)

[16] Arnaldo García and Henning Stichtenoth, A tower of Artin-Schreier extensions of function fields attaining the Drinfel' $d$-Vlădut bound, Invent. Math. 121 (1995), no. 1, 211-222, DOI 10.1007/BF01884295. MR1345289(96d:11074)

[17] Arnaldo Garcia, Henning Stichtenoth, and Hans-Georg Rück, On tame towers over finite fields, J. Reine Angew. Math. 557 (2003), 53-80, DOI 10.1515/crll.2003.034. MR.1978402 (2004e:11133)

[18] Hugues Randriambololona, Bilinear complexity of algebras and the ChudnovskyChudnovsky interpolation method, J. Complexity 28 (2012), no. 4, 489-517, DOI 10.1016/j.jco.2012.02.005. MR2925903

[19] Mohammad Amin Shokrollahi, Optimal algorithms for multiplication in certain finite fields using elliptic curves, SIAM J. Comput. 21 (1992), no. 6, 1193-1198, DOI 10.1137/0221071. MR.1192302 (93m:11138)

[20] Igor E. Shparlinski, Michael A. Tsfasman, and Serge G. Vladut, Curves with many points and multiplication in finite fields, Coding theory and algebraic geometry (Luminy, 1991), Lecture Notes in Math., vol. 1518, Springer, Berlin, 1992, pp. 145-169, DOI 10.1007/BFb0087999. MR:1186422(93h:11063)

[21] S. Winograd, On multiplication in algebraic extension fields, Theoret. Comput. Sci. 8 (1979), no. 3, 359-377, DOI 10.1016/0304-3975(79)90017-3. MR532476 (80e:68081)

Inria Saclay, LiX, École Polytechnique, 91128 Palaiseau Cedex, France

E-mail address: pieltant@lix.polytechnique.fr

ENST ("Telecom Paristech"), 46 rue Barrault, F-75634 Paris Cedex 13, France

E-mail address: randriam@telecom-paristech.fr 